\title{
CONTENIDOS, METODOLOGÍA Y ALUMNADO. UNA VALORACIÓN CRÍTICA DE LA ENSEÑANZA EN BUP Y COU
}

\author{
AFONSO LÓPEZ, R. ${ }^{1}$, BAZO GONZÁLEZ, C. ${ }^{2}$, HENRÍQUEZ SANTANA, J.A. ${ }^{3}$, \\ LÓPEZ HERNÁNDEZ, M. ${ }^{4}$, MACAU FÁBREGA, M.D. ${ }^{5}$, MARRERO ACOSTA, J. ${ }^{6} y$ \\ RODRÍGUEZ PALMERO, M.L.? \\ 1 Instituto de Bachillerato Tomás de Iriarte. Santa Cruz de Tenerife. \\ 2 Centro de Enseñanzas Integradas. La Laguna. Santa Cruz de Tenerife. \\ ${ }^{3}$ Instituto de Bachillerato Teobaldo Power. Santa Cruz de Tenerife. \\ ${ }^{4}$ Instituto de Bachillerato Mencey Acaymo. Güimar. Santa Cruz de Tenerife. \\ 5 Inspección de Enseñanza Media. Santa Cruz de Tenerife. \\ - Departamento de Didáctica e Investigación Educativa y del Comportamiento. \\ Universidad de La Laguna. Santa Crux de Tenerife. \\ 7 Instituto de Bachillerato Dr. A. González y González. Tejina. La Laguna. \\ Santa Cruz de Tenerife.
}

\section{SUMMARY}

The aim of this study was to compare the interest of the students in Natural Science in front of three other subjects such as Mathematics, Literature and Geography \& History. The selected items of the contents of every subject, and the importance of teaching as an influential factor on students were also studied. The results showed that both the selection of the contents and the methods should have been modified.

\section{INTRODUCCIÓN}

Los procesos de enseñanza y de aprendizaje han acaparado en los últimos tiempos la atención de una gran cantidad de investigadores cuyos orígenes son diferentes, como diferentes son también las perspectivas de análisis sobre las que estudiarlos. Desde la psicología, la pedagogía, la didáctica general o las específicas, son muchos los elementos que se han incorporado a este sistema aula-enseñante-enseñados, elementos que constituyen, en definitiva, lo que conocemos como teoría curricular. Quizás esto no sea más que una moda o, más bien, un intento fundamentado de lo que plantean Boyer y Tiberghien:

Actualmente la formación científica está en cuestión en numerosos paises. Los trabajos de investigación la abordan de diversas formas, pero privilegian el saber en juego en la enseñanza. Unos consideran su apropiación por los alumnos; otros, la forma en que se introducen en la clase, los libros, los programas; otros, en la medida que es ocasión de interacción entre dos actores: profesor y alumno. [...]. También son objeto de investigación el interés, la motivación, la actitud de los alumnos [...], la imagen de la ciencia [...] (Boyer y Tiberghien, 1989, pp. 213-214).

Son, como decíamos, muchas las posibilidades, muchos los intentos de buscar mejores modos de hacer que la enseñanza y el aprendizaje sean más eficaces.

A la hora de definir esos procesos, cualquier evento educativo implica una acción para intercambiar significados y sentimientos entre el profesor y el alumno. (Moreira, 1993, p. 2). En este sentido, los procesos de enseñanza y de aprendizaje son procesos de negociación 
de significados, de construcción progresiva de conocimientos «científicos» en la medida en que se refieren a conocimientos más aceptados, más compartidos (Moreira, 1993). Por ello, cuando hablamos de «conocimiento científico», estaremos haciendo referencia a todo aquel conocimiento que se ha construido y que pretendemos negociar con nuestro alumnado, independientemente de la disciplina de que se trate. Nadie cuestionaría en este momento que se ha producido una ampliación importante de los límites de lo que se considera el objeto de la ciencia, que es evidente que no se circunscribe sólo a lo que tradicionalmente se ha considerado disciplina científica (manipulativa, experimental, etc.) y que esta «nueva» consideración tiene importantes consecuencias en la enseñanza (Moreno, 1993).

En la línea expuesta, y aunque la preocupación por el profesorado como uno de los factores esenciales del proceso de enseñanza-aprendizaje es antigua [...], hasta recientemente los estudios se centraban en las características del buen profesor o en las diferencias entre buenos y malos profesores [...], mientras que ahora la cuestión se plantea en términos de cuáles son los conocimientos que los profesores necesitamos adquirir. El matiz es sin duda importante y supone una superación de concepciones esencialistas ("se es», "se nace», [...] buen profesor) que apuntaban a ineficaces políticas de selección más que a procesos de formación. Debemos congratularnos, pues, de dicha evolución y plantearnos cuales son los conocimientos que precisamos adquirir para desarrollar adecuadamente nuestra actividad docente (Gil, 1991, p. 70).

Esto supone un proceso continuo de revisión que necesariamente tendrá que estar centrado en la reflexión sobre la práctica diaria y que, por tanto, requerirá instrumentos adecuados para su evaluación. En este sentido, y como una de esas herramientas, tiene un significado especial conocer lo que nuestro alumnado opina, lo que vive o lo que siente en relación con nuestro trabajo, pues nuestra visión, como docentes, sobre el mismo hecho educativo es diferente a la que aquél percibe, dado que se ve influida por lo que se ha dado en llamar «pensamiento implícito del profesorado». De acuerdo con Carrascosa y sus colaboradores (1991, p. 46): Esto no solo reforzarfa la validez de las propuestas de cambio didáctico elaboradas, sino que permitiría incluso utilizar la valoración de los alumnos como uno de los instrumentos de evaluación de dicho cambio.

Podría pensarse que, efectivamente, las opiniones del alumnado no son fiables, que no sirven para cubrir esta finalidad, ya que la carga de subjetividad que podrían soportar es muy grande. Muy al contrario, consideramos que es muy importante tener esta información y lo es si nosotros como profesores [...] no hacemos aquello que es apropiado, y lo sigue siendo, si, a pesar de nuestro convencimiento de estar enseñando apropiadamente, ninguno to nota (Carrascosa et al., 1991, p. 51).

Partiendo de este convencimiento, nos interesa saber cómo valoran los alumnos algunos de los conocimien- tos, destrezas y actitudes que, según la investigación, convendría que los profesores y profesoras poseyeran. Ello puede contribuir a un mejor diseño de las estrategias de cambio didáctico, puesto que un comportamiento efectivo del profesorado supone su aceptación por los alumnos (Carrascosa et al., 1991, p. 46).

Schwab (1973) planteaba que cualquier fenómeno educativo implica cuatroelementos(lugares comunes): aprendiz (aprendizaje), profesor (enseñanza), materia de enseñanza (currículo) y matriz social (medio, contexto). En aras de definir con mayor claridad aquello que influye directamente en esos fenómenos educativos, podrían definirse cinco elementos: aprendiz, profesor, conocimiento, contexto y evaluación (Novak, 1977; Moreira. 1993). Todos y cada uno de estos elementos tienen implicaciones fundamentales y decisivas en los procesos generados en el aula. Centramos nuestra atención en el estudiante (como protagonista de la investigación), el profesor (en la medida en que obtendremos información sobre sus formas de trabajo) y en el conocimiento (materia de enseñanza), pues su selección se ve cuestionada. Indirectamente, contexto y evaluación (los elementos restantes) también se ven reflejados, en la medida en que se está utilizando la opinión del alumnado como instrumento de recogida de información para analizar los contextos educativos desde una óptica peculiar y poco frecuente como es la de los jóvenes.

Si bien el aprendizaje de las ciencias (en sentido estricto) está resultando insuficiente, como muestran las investigaciones que veremos, y se ve influido por multitud de factores, de entre los cuales, Ios relativos a la actitud del alumnado, el ambiente del aula, etc. son elementos fundamentales, parece ser que hay una cierta mayor aceptación de las ciencias naturales en relación con otras disciplinas con las que se ha comparado (matemáticas, lengua y literatura, y ciencias sociales), por parte de los estudiantes (Serrano, 1988; Afonso López et al., 1991; Acevedo Díaz, 1993). Las razones de esta diferencia son las que se han constituido en objeto de nuestra investigación, en primer lugar, por la novedad del dato y, en segundo Iugar, porque la bibliografía consultada no ofrece explicaciones de esta diferencia. Cabe la posibilidad, en nuestra opinion, de que dicha diferencia esté relacionada con la selección que se ha hecho de los contenidos que se imparten en cada una de estas asignaturas, por una parte, $y$, por otra, con la posibilidad de que existan planteamientos metodológicos diferentes, distintas formas de trabajar esos contenidos en el aula, que puedan ser incluso idiosincráticos de cada materia. Por esta razón, considerando como elemento fundamental de evaluación al propio alumnado, se ha desarrollado una investigación que permite contrastar las hipótesis planteadas. Éstas son:

1. Las ciencias naturales son más interesantes para el alumnado que otras materias por el contenido seleccionado.

2. Las ciencias naturales son más aceptadas y valoradas que otras materias por el uso de una metodología más innovadora en su enseñanza. 
Tabla I

Cuadro esquemático de las investigaciones consultadas.

\begin{tabular}{|c|c|c|c|c|}
\hline AUTOR. AÑO & OBJETO & MUESTRA & INSTRUMENTO & RESULTADOS PRINCIPALES \\
\hline $\begin{array}{l}\text { Yager y Penick } \\
1984\end{array}$ & $\begin{array}{l}\text { Papel de algunos elementos } \\
\text { no directamente } \\
\text { relacionados } \\
\text { con la adquisición de } \\
\text { relaciones profesorado/ } \\
\text { alumnado así } \\
\text { como actitudes del } \\
\text { alumnado frente a la } \\
\text { ciencia. }\end{array}$ & $\begin{array}{l}2.500 \\
\text { estudiantes } \\
\text { de } 13 \text { y } 17 \\
\text { años. }\end{array}$ & Cuestionario. & $\begin{array}{l}\text { * Poco interés del profesorado por el } \\
\text { alumnado. } \\
\text { * El alumnado no participa en la } \\
\text { selección del contenido. }\end{array}$ \\
\hline $\begin{array}{l}\text { Yager y Penick } \\
1986\end{array}$ & $\begin{array}{l}\text { Actitudes y percepciones de } \\
\text { los estudiantes sobre la } \\
\text { ciencia, sus docentes y el } \\
\text { valor del conocimiento } \\
\text { cientifico. }\end{array}$ & $\begin{array}{l}750 \text { estudiantes } \\
\text { de } 9,13 \text { y } 17 \\
\text { años y } 310 \\
\text { jovenes que } \\
\text { han abandonado } \\
\text { la escuela. }\end{array}$ & Cuestionario. & $\begin{array}{l}\text { * Disminución de la valoración positiva } \\
\text { hacia la ciencia con la edad. } \\
\text { * Imagen del profesorado más negativa } \\
\text { en aumento con la edad del alumado. } \\
\text { * Necesidad de cambiar la metodologia, } \\
\text { la estrategia, la selección de contenidos } \\
\text { y las expectativas. }\end{array}$ \\
\hline $\begin{array}{l}\text { Talton y Simpson } \\
1986\end{array}$ & $\begin{array}{l}\text { Influencia de la personalidad } \\
\text { de los jovenes, ia familia y } \\
\text { el ambiente en las actitudes. }\end{array}$ & $\begin{array}{l}\text { Alumnado de } \\
11 \text { a } 16 \text { años } \\
\text { de } 12 \\
\text { escuelas. }\end{array}$ & Cuestionario. & $\begin{array}{l}\text { * Las variables relacionadas con el } \\
\text { ambiente educativo son las que mayor } \\
\text { influencia tienen en la actitud del } \\
\text { alumnado. } \\
\text { * El ambiente de clase está bajo control } \\
\text { del educador. }\end{array}$ \\
\hline $\begin{array}{l}\text { Schibecci y Riley } \\
1986\end{array}$ & $\begin{array}{l}\text { Variables que afectanal alum- } \\
\text { nado, sus percepciones de la } \\
\text { ciencia y su influencia en los } \\
\text { resultados académicos. }\end{array}$ & $\begin{array}{l}\text { Base de datos } \\
\text { de NAEP. } \\
(1976-77)\end{array}$ & Cuestionario. & $\begin{array}{l}\text { * Existe relación causal sustancial entre } \\
\text { actitudes y resultados académicos. } \\
\text { * Las percepciones de los estudiantes de } \\
\text { la ensentanza influyen en sus actitudes. }\end{array}$ \\
\hline $\begin{array}{l}\text { Schibecci } \\
1986\end{array}$ & $\begin{array}{l}\text { Actitudes hacia la ciencia y su } \\
\text { aprendizaje. }\end{array}$ & & $\begin{array}{l}\text { - Revisión de } \\
\text { cómics, novelas y } \\
\text { otros mass media. } \\
\text { - Estudio de } \\
\text { actitudes de } \\
\text { estudiantes y } \\
\text { público en general. }\end{array}$ & $\begin{array}{l}\text { * Las clases de ciencias no consiguen } \\
\text { transmitir una imagen coherente de los } \\
\text { investigadores y de su trabajo. }\end{array}$ \\
\hline $\begin{array}{l}\text { Arana y otros } \\
1987\end{array}$ & $\begin{array}{l}\text { Imagen de las asignaturas de } \\
\text { ciencias y su evolucion en } \\
\text { dificultad, importancia y } \\
\text { amenidad. }\end{array}$ & $\begin{array}{l}1.606 \text { jóvenes de } \\
8^{\circ} \text { de EGB, } \\
1^{\circ} \text { y } 3^{\circ} \text { de BUP, } \\
1^{\circ} \text { de FP-I y } \\
1^{\circ} \text { de FP-II. }\end{array}$ & Cuestionarios. & $\begin{array}{l}\text { * Las ciencias reciben consideración } \\
\text { intermedia en las tres dimensiones. }\end{array}$ \\
\hline $\begin{array}{l}\text { Serrano } \\
1988\end{array}$ & $\begin{array}{l}\text { Estudio de la actitud hacia el } \\
\text { aprendizaje de las ciencias y } \\
\text { hacia la dimension social de } \\
\text { las mismas. }\end{array}$ & $\begin{array}{l}80 \text { estudiantes } \\
\text { de distintos } \\
\text { centros. }\end{array}$ & $\begin{array}{l}\text { Diferencial } \\
\text { semántico. }\end{array}$ & $\begin{array}{l}\text { * Valoración más favorable hacia } \\
\text { las ciencias. } \\
\text { * Las ciencias son más interesantes } \\
\text { para el alumnado. } \\
\text { * Se mantiene el interés por las } \\
\text { ciencias con el tiempo. } \\
\text { * La escasez de trabajos prácticos } \\
\text { no parece influir en ese interés. }\end{array}$ \\
\hline
\end{tabular}


Tabla I (continuación)

\begin{tabular}{|c|c|c|c|c|}
\hline AUTOR, AÑO & OBJETO & MUESTRA & INSTRUMENTO & RESULTADOS PRINCIPALES \\
\hline $\begin{array}{l}\text { Simpson y Oliver } \\
\text { I990 }\end{array}$ & $\begin{array}{l}\text { Distintas influencias que se } \\
\text { pueden ejercer sobre la } \\
\text { actitud de los estudiantes } \\
\text { frente al aprendizaje de las } \\
\text { ciencias. }\end{array}$ & $\begin{array}{l}4.500 \\
\text { estudiantes. } \\
78 \text { docentes. }\end{array}$ & $\begin{array}{l}\text { * Cuestionario de } \\
\text { actitudes. } \\
\text { * Resultados de } \\
\text { exámenes. } \\
\text { * Cuestionarios } \\
\text { para caracteris- } \\
\text { ticas profesio- } \\
\text { nales y actitudes } \\
\text { de docentes. }\end{array}$ & $\begin{array}{l}\text { * Poco interés del profesorado por el } \\
\text { alumnado. } \\
\text { * El alumnado no participa en la } \\
\text { selección del contenido. } \\
\text { * Disminución de la actitud positiva } \\
\text { hacia la ciencia a lo largo del curso. } \\
\text { * Disminución de la motivación para } \\
\text { obtener buenos resultados a medida que } \\
\text { avanza el curso. } \\
\text { * Mayor descenso de las actitudes en } \\
\text { grupos unormales». Infuencia positiva } \\
\text { de mayor dedicacion del profesorado en } \\
\text { los otros grupos. } \\
\text { * Los factores relativos al aula } \\
\text { (clima, actitudes y comporamiento del } \\
\text { docente) son los que ejercen la mayor } \\
\text { influencia en la actitud del alumnado. }\end{array}$ \\
\hline $\begin{array}{l}\text { Fontes Costa } \\
1990\end{array}$ & $\begin{array}{l}\text { Representación del alum- } \\
\text { nado sobre la realización de } \\
\text { actividades de aprendizaje } \\
\text { relacionadas con el dominio } \\
\text { socioafectivo, } \\
\text { asi como la influencia de las } \\
\text { mismas en su aprendizaje } \\
\text { y en la evaluación. }\end{array}$ & $\begin{array}{l}152 \\
\text { estudiantes } \\
\text { entre } 14 \text { y } 20 \\
\text { ấos. }\end{array}$ & Cuestionario. & $\begin{array}{l}\text { * Las actividades que permiten el } \\
\text { desarrollo de actitudes y valores se } \\
\text { realizan en un mínimo muy reducido } \\
\text { y son poco variadas. } \\
\text { * Los estudiantes consideran que estos } \\
\text { elementos no deben ser evaluados } \\
\text { dentro del rendimiento escolar. }\end{array}$ \\
\hline $\begin{array}{l}\text { Afonso López } \\
\text { y otros. } 1991\end{array}$ & $\begin{array}{l}\text { Intereses, actitudes, } \\
\text { dificultades y opiniones del } \\
\text { alumnado en relación con la } \\
\text { enseñanza de las ciencias. }\end{array}$ & $\begin{array}{l}394 \text { y } 346 \\
\text { estudiantes de } \\
1^{\circ} \text { BUP, } \\
3^{\circ} \text { BUP } \\
\text { y COU. }\end{array}$ & Cuestionarios (2). & $\begin{array}{l}\text { * CCNN es la asignatura más } \\
\text { interesante. } \\
\text { * MAT y LL son las asignaturas más } \\
\text { útiles para el alumnado en diferentes } \\
\text { contextos. } \\
\text { * La metodología utilizada en el aula } \\
\text { resulta cuestionada, siendo más aceptada } \\
\text { la aplicada en CCNN. } \\
\text { * Se asigna un alto grado de } \\
\text { responsabilidad al profesorado. }\end{array}$ \\
\hline $\begin{array}{l}\text { Carrascosa y otros } \\
199]\end{array}$ & $\begin{array}{l}\text { Vision del alumnado sobre } \\
\text { el conocimiento que deberia } \\
\text { tener el profesorado de } \\
\text { ciencias. }\end{array}$ & $\begin{array}{l}\text { Estudiantes } \\
\text { de } 2^{\circ} \mathrm{BUP} \\
3^{\circ} \mathrm{BUP} \text { y } \\
\text { COU de } \\
\text { cuatro centros. }\end{array}$ & Cuestionarios (2). & $\begin{array}{l}\text { * El alumnado de enseñanza media } \\
\text { percibe los aspectos importantes o } \\
\text { esenciales del conocimiento profesional } \\
\text { de sus docentes. } \\
\text { * El profesorado de } \mathrm{F} \text { y } \mathrm{Q} \text { no desarrolla } \\
\text { en sus clases la mayoria de esos } \\
\text { aspectos. } \\
{ }^{*} \text { La imagen negativa se agrava a } \\
\text { medida que avanza eI nivel educativo. } \\
\text { * El resultado es significativamente } \\
\text { positivo para docentes implicados en la } \\
\text { investigación educativa. }\end{array}$ \\
\hline $\begin{array}{l}\text { Espinosa y Román } \\
\text { I991 }\end{array}$ & $\begin{array}{l}\text { Influencia de las actitudes } \\
\text { hacia la ciencia y de las } \\
\text { asignaturas pendientes en el } \\
\text { rendimiento en ciencias. }\end{array}$ & $\begin{array}{l}393 \text { estudiantes } \\
\text { de } 1^{\circ} \text { BUP } \\
\text { y } 394 \\
\text { de } 2^{\circ} \text { BUP. }\end{array}$ & $\begin{array}{l}\text { * Cuestionario } \\
\text { (diferencial } \\
\text { semśntico). } \\
\text { * Calificaciones } \\
\text { de Fy Q de sunio. }\end{array}$ & $\begin{array}{l}\text { * Actitud negativa del alumnado } \\
\text { frente al estudio de F y Q. } \\
\text { * Se detecta influencia negativa } \\
\text { cuantiffcable de las astgnaturas } \\
\text { pendientes en el rendimiento. }\end{array}$ \\
\hline $\begin{array}{l}\text { Ortega Ruiz } \\
\text { y otros. I992 }\end{array}$ & $\begin{array}{l}\text { Diseño y aplicación de una } \\
\text { escala de actitudes hacia el } \\
\text { estudio de las ciencias } \\
\text { experimentales. }\end{array}$ & $\begin{array}{l}57 \text { y } 54 \\
\text { estudiantes de } \\
\text { FP de dos } \\
\text { centros. }\end{array}$ & Cuestionario. & $\begin{array}{l}\text { * El alumnado manifiesta rechazo hacia } \\
\text { las ciencias. } \\
\text { "Este rechazo aumenta a medida que se } \\
\text { estudian más cursos escolares. }\end{array}$ \\
\hline
\end{tabular}


Tabla I (continuación)

\begin{tabular}{|c|c|c|c|c|}
\hline AUTOR AÑO & OBJETO & MUESTRA & INSTRUMENTO & RESULTADOS PRINCIPALES \\
\hline $\begin{array}{l}\text { Espinosa y Román } \\
1993\end{array}$ & $\begin{array}{l}\text { Actitudes de estudiantes } \\
\text { universitarios hacia la } \\
\text { ciencia. }\end{array}$ & $\begin{array}{l}240 \\
\text { estudiantes } \\
\text { de estudios } \\
\text { cientificos. }\end{array}$ & Cuestionario. & $\begin{array}{l}\text { Evolución de la actitud hacia la ciencia } \\
\text { «en diente de sierra» y no lineal. }\end{array}$ \\
\hline $\begin{array}{l}\text { Acevedo Díaz } \\
1993\end{array}$ & $\begin{array}{l}\text { Opinión del alumnado sobre } \\
\text { la ciencia desde las } \\
\text { relaciones CTS. }\end{array}$ & $\begin{array}{l}220 \\
\text { estudiantes } \\
\text { de } 2^{\circ} \text { BuP } \\
\text { y COU. }\end{array}$ & Cuestionario. & $\begin{array}{l}\text { * Neutralidad ideológica de la ciencia } \\
\text { y de los científicos. } \\
\text { * Imagen distorsionada de los científicos. } \\
\text { * Escasa e inadecuada incidencia del } \\
\text { contenido para superar ideas no } \\
\text { aceptadas actualmente. }\end{array}$ \\
\hline $\begin{array}{l}\text { Acevedo Díaz } \\
1993\end{array}$ & $\begin{array}{l}\text { Anślisis de actitudes } \\
\text { hacia el apiendizaje de } \\
\text { la F, Q, B, G y M frente } \\
\text { a amenidad, dificultad } \\
\text { e importancia social. }\end{array}$ & $\begin{array}{l}220 \\
\text { estudiantes } \\
\text { de } 2^{\circ} \text { BUP } \\
\text { y COU. }\end{array}$ & $\begin{array}{l}\text { Cuestionario } \\
\text { (Diferencial } \\
\text { semántico). }\end{array}$ & $\begin{array}{l}\text { * Las CCNN son para } 2^{\circ} \text { BUP amenas e } \\
\text { importantes socialmente. } \\
{ }^{*} \text { La actitud global es positiva en COU } \\
\text { solo hacia la } B \text {, que es amena. } \\
{ }^{*} \text { La } G \text { no tiene importancia social. }\end{array}$ \\
\hline
\end{tabular}

Las referencias bibliográficas consultadas no ofrecen investigaciones y experiencias seguidas en este sentido, si bien es cierto que nos han permitido interpretar algunos de los datos obtenidos en el presente trabajo. Estas referencias son las que se presentan a continuación:

Yager y Penick (1984) han determinado el papel de algunos elementos no directamente relacionados con la adquisicion de conocimiento como, por ejemplo, la relación profesorado-alumnado; estos mismos autores (Yager y Penick, 1986) han trabajado las actitudes y percepciones de los estudiantes de ciencias, en sentido clásico, sobre las clases, los profesores y el valor relativo de los estudios científicos; Talton y Simpson (1986) hacen una investigación sobre la influencia de la personalidad de los jóvenes, la familia y el ambiente en la actitud de Ios estudiantes; Schibecci y Riley (1986) analizan las variables que afectan a los estudiantes, sus percepciones de la ciencia, tradicionalmente considera$\mathrm{da}$, y la influencia que esto tiene en los resultados académicos; Schibecci (1986) estudia las actitudes hacia la ciencia y su aprendizaje. En este contexto, resultan de interés también: los trabajos de Arana y sus colaboradores (1987) sobre dificultad, importancia y amenidad de la ciencia; el estudio sobre actitudes desarrollado por Serrano (1988); los trabajos de Simpson y Oliver (1990) sobre las distintas influencias que pueden ejercerse sobre la actitud de los estudiantes frente al aprendizaje de las ciencias; Ios trabajos desarrollados por Fontes Costa (1990), en los que el alumnado manifiesta que las actividades que permiten el desarrollo de actitudes y valores se realizan en un mínimo muy reducido y son poco variadas. Por otra parte, estos mismos estudiantes los consideran elementos que no deben ser evaluados dentro del rendimiento escolar; los intereses, actitudes, dificultades y opiniones del alumnado de BUP y COU en relación con la enseñanza de las ciencias naturales, llevado a cabo por Afonso López y otros (1991); la investigación desarroliada por Carrascosa y otros (1991) sobre lo que el profesorado debe saber y saber hacer en las clases de ciencias ( $\mathrm{experimentales!);} \mathrm{la} \mathrm{investiga-}$ ción llevada a cabo por Ortega Ruiz y sus colaboradores (1992) en la que el alumnado de formación profesional manifiesta rechazo hacia las ciencias, rechazo que aumenta a medida que se estudian más cursos escolares; las tendencias observadas en los trabajos longitudinales llevados a cabo por Espinosa y Román (I991, 1993) sobre las actitudes hacia las ciencias, relativas a diferentes aspectos y niveles; los datos obtenidos por Acevedo Díaz (1993), enfocados hacia la amenidad, la dificultad y la importancia social de diferentes ciencias.

Los rasgos más relevantes de los trabajos comentados figuran en la tabla I.

Como se ve, se trata de investigaciones que tienen como protagonistas directos a los estudiantes en diferentes aspectos. No se han encontrado referencias centradas directamente en la selección del contenido y en la forma de contemplar la metodología; tampoco se ha podido disponer de trabajos comparativos de distintas asignaturas.

\section{MÉTODO}

\section{Sujetos}

El estudio que presentamos se ha llevado a cabo sobre una muestra que afecta a 1.546 estudiantes de $1^{\circ}$ de BUP y de COU, tanto de ciencias como de letras, de la isla de 
Tabla II

Distribución del alumnado encuestado.

\begin{tabular}{|c|c|c|c|c|}
\hline & $1^{\circ}$ de BUP & CoU-Letras & CoU-Ciencias & Total \\
\hline IB-A & 236 & 127 & 227 & 590 \\
\hline IB-B & 202 & 116 & 109 & 427 \\
\hline IB-C & 113 & 208 & 208 & 529 \\
\hline TOTAL & 551 & 451 & 544 & 1546 \\
\hline
\end{tabular}

Tenerife, lo que representa el $22,27 \%$ del total del alumnado de estos niveles educativos. La encuesta se distribuyó entre los implicados durante el curso escolar 1991-92 en tres centros distintos de bachillerato: uno de la zona norte (IB-A), otro de la zona sur (IB-B), ambos rurales, $y$ un tercer centro que hemos considerado urbano, cuyas características se corresponden con las de los denominados capitalinos (IB-C). Una vez seleccionados los centros, elegidos de esta manera con objeto de analizar las posibles diferencias (comarcales y urbanorurales), se optó por determinar la muestra, de manera totalmente aleatoria, cuya distribución responde a lo expresado en la tabla II.
Este alumnado estaba adscrito a cuarenta grupos naturales o habituales de clase. La muestra de estudiantes seleccionados representa un total de 160 profesores de las distintas asignaturas objeto de nuestro estudio.

\section{Instrumento}

El instrumento utilizado para obtener la información que se necesitaba ha sido un único cuestionario que, para responder a las dos hipótesis formuladas, constaba de dos partes:

Tabia IIl

Relación de temas por asignatura.

\begin{tabular}{|c|c|c|c|}
\hline MATEMÁTICAS & LENGUA Y LITERATURA & CIENCIAS NATURALES & CIENCIAS SOCIALES \\
\hline $\begin{array}{l}\text { Combinatoria y } \\
\text { probabilidad }\end{array}$ & $\begin{array}{l}\text { Análisis de } \\
\text { textos }\end{array}$ & $\begin{array}{l}\text { Agentes geologicos } \\
\text { extemos }\end{array}$ & $\begin{array}{l}\text { Capitalismo } \\
\text { Socialismo }\end{array}$ \\
\hline Estadística & $\begin{array}{l}\text { Análisis morfo- } \\
\text { sintáctico }\end{array}$ & Ecologia & Edad Media \\
\hline $\begin{array}{l}\text { Funciones y } \\
\text { graficas }\end{array}$ & $\begin{array}{l}\text { Creación } \\
\text { literaria }\end{array}$ & $\begin{array}{l}\text { La célula y su } \\
\text { funcionamiento }\end{array}$ & El Renacimiento \\
\hline Geometría & Escenificación & $\begin{array}{l}\text { Minerales y } \\
\text { rocas }\end{array}$ & Revoluciones \\
\hline $\begin{array}{l}\text { Números y } \\
\text { operaciones }\end{array}$ & Expresión oral & Seres vivos & $\begin{array}{l}\text { Sectores } \\
\text { económicos }\end{array}$ \\
\hline $\begin{array}{l}\text { Sistemas de } \\
\text { ecuaciones }\end{array}$ & Lecturas & $\begin{array}{l}\text { Tectónica de } \\
\text { placas }\end{array}$ & $\begin{array}{l}\text { Sistema socio- } \\
\text { político griego }\end{array}$ \\
\hline
\end{tabular}


- Una, dirigida al análisis de la primera de las hipótesis; para ello se han planteado una serie de seis grandes temas relativos a cada una de las asignaturas objeto de estudio, con la finalidad de que los estudiantes los puntuaran de 0 a 5 , según el interés que en ellos despertaban. Los temas seleccionados en las cuatro asignaturas han sido los que se exponen en la tabla III.

La elección de estos seis grandes núcleos se ha llevado a cabo a través de una serie de consultas a distintos profesionales o expertos. A priori se consideraron una muestra representativa del contenido que se imparte en cada una de ellas, y lo suficientemente genérica como para que pudiera ser válida para los tres niveles encuestados.

Esta primera parte del cuestionario permite no sólo valorar la asignatura o asignaturas cuyos contenidos, globalmente entendidos, son los que despiertan el mayor interés en el alumnado, sino también analizar, dentro de cada una de ellas, cuáles son los temas o núcleos que reciben las mayores puntuaciones; esta última cuestión cobra una especial significación en relación con la selección del contenido que el profesorado lleva a cabo en sus programaciones.

- La otra parte pretendía recabar información relativa a la segunda de las hipótesis. Para ello se han redactado catorce ítems centrados en los diferentes elementos que, teóricamente, pueden y deben considerarse en relación con la metodología. De estos catorce ítems, dos se refieren a estrategias y, del resto, tres están dirigidos a cada uno de los elementos restantes (actividades y tareas, papel del profesorado, medios y recursos, y entorno del aprendizaje). Para cada uno de ellos se han buscado frases representativas que denotan diferentes prácticas docentes. Estos ítems se han distribuido al azar, con objeto de que no quedaran contiguos los relativos a un mismo elemento. En cada uno de ellos, los estudiantes se situaban en una escala de cuatro niveles, según la frecuencia con la que, en su opinión, la frase en cuestión representaba la práctica diaria de cada una de las asignaturas. La agrupación teórica de los distintos ítems destinados a Ia metodología ha sido corroborada por el análisis matemático de los mismos a través de un estudio de correlación (Anexo I).

Como se ve, se han confeccionado cuestionarios cerrados para ambas partes o aspectos objeto de estudio (uno para caca asignatura), porque se consideró que resultaban más operativos en una doble vertiente: por una parte, para el alumnado que los cumplimentaba y, por otra, para el análisis de los datos que se obtuvieran. En el tratamiento de este cuestionario se ha estimado estadísticamente significativo el $99 \%$. El coeficiente de fiabilidad aplicado ha sido Alpha de Cronbach; el valor obtenido para el mismo es de 0,54. Las comparaciones de los valores medios se han hecho con $t$ de student. La información obtenida se ha tratado, en primer lugar, atendiendo a cada una de las partes o aspectos que hemos definido en el cuestionario y, en segundo lugar, al estudio de regresión múttiple que se ha llevado a cabo con objeto de relacionarlas; los porcentajes que se presentan se han calculado a través del índice que, sobre el total, representa cada uno de los elementos de la metodología, utilizando díchos coeficientes de regresión múltiple.

\section{Procedimiento}

El estudio que se presenta es el fruto de un trabajo que se ha desarrollado en diferentes fases, la primera de las cuales se centró en la delimitación de las posibles explicaciones a la mayor aceptación, en líneas generales, como hemos comentado, de las ciencias naturales en relación con las tres asignaturas con las que se ha comparado. Una vez aclarado este extremo se discutieron en el seno del grupo ANAMBRO distintas posibilidades para obtener la información, partiendo, como premisa, de la idea de que tenía que ser un cuestionario cerrado (por las razones ya expuestas) que no fuera excesivamente largo. Después de varias reuniones dedi. cadas a esta tarea, y con asesoramiento específico de expertos, se optó por elaborar el instrumento ya descrito, estableciendo cuatro modelos (uno por cada asignatura).

Una segunda fase se centró en la recogida đe la información, en definitiva, en que los estudiantes cumplimenta. ran la encuesta. Como ya se ha comentado, se pasó durante el curso escolar 199 I „92; los cuestionarios de las distintas asignaturas se han entregado al azar y en periodo normal de clase, procurando que no fuera ni en los primeros ni en los úlitimos meses, tiempos estos en los que las opiniones podían estar desvirtuadas por desconocimiento (al principio) o por actitudes negativas (al final).

Se contó con la colaboración de diferentes docentes, dada la imposibilidad de que entregaran el cuestionario y estuvieran presentes directamente las personas integrantes del grupo. Si bien se quiere dejar constancia de nuestro agradecimiento, pues sin esta ayuda el trabajo no hubiese sido posible, se constata que surgen mayores dificultades de comprensión y de aplicación de una encuesta si no son sus propios diseñadores los que la aplican directamente.

Como tercera fase, dentro del procedimiento seguido, se ha centrado la atención en la elaboración de las categorías que permitieran el análisis de los resultados. En la medida en que se trata de un cuestionario cerrado, puede considerarse que, casi en su totalidad, estas categorías se han establecido a priori. En la primera parte, lo que se hizo necesario fue establecer la consideración de que se aparejaba a cada una de las puntuaciones posibles, obteniéndose la tipificación siguiente:
0 - Ningún interés
1 - Algún interés (apenas)
2- Poco interés
3 - Bastante interés
4- Mucho interés
5 - Máximo interés

En la segunda parte de la encuesta, en relación con esta fase, lo que se hizo fue reunir los f́tems relativos a cada 
uno de los elementos de la metodología tratados. La categorización de las respuestas se había hecho explícita en el propio cuestionario, estableciéndose una escala de cuatro niveles:

$$
\begin{aligned}
& \text { 0- Nada o nunca } \\
& \text { 1- Poco } \\
& \text { 2- Bastante } \\
& \text { 3- Mucho o siempre }
\end{aligned}
$$

\section{RESULTADOS}

Para hacer la presentación de los resultados, seguiremos el mismo esquema que el que ha servido de guía, tanto en la formulación de las hipótesis, como en el cuestionario que pretende contrastarlas. Por ello, se dedica un primer apartado a analizar la información obtenida en relación con el interés del alumnado, y un segundo apartado para estudiar las consideraciones que hace el mismo sobre la metodología que los docentes de las distintas asignaturas trabajamos en las aulas; un tercer apartado se centra en la relación entre ambas partes o aspectos.

\section{A. Interés del alumnado}

Dado que los datos obtenidos son muchos, y con objeto de que sea utilizable la información que se deriva de ellos, se ha optado por organizar ésta en torno a cuatro aspectos diferentes, dependiendo de las variables consideradas.

\section{A.1. Interés global por asignaturas}

Las medias totales que manifiestan los estudiantes, en relación con las cuatro asignaturas objeto de estudio son las siguientes (Tabla IV):

Tabla IV

Valores medios globales por asignatura.

\begin{tabular}{|l|c|c|}
\hline Asignatura & $\overline{\mathrm{X}}$ & $\sigma$ \\
\hline MATEMÁTICAS & 2,83 & 0,87 \\
\hline LENGUA Y LITERATURA & 3,00 & 0,84 \\
\hline CIENCIAS NATURALES & 2,95 & 0,81 \\
\hline CIENCIAS SOCIALES & 2,72 & 0,88 \\
\hline
\end{tabular}

Este resultado, por sí sólo, considerando toda la información de modo conjunto, no refleja diferencias significativas entre los intereses manifestados por el alumnado, en relación con los temas seleccionados para el cuestio nario. Llama la atención el hecho de que los estudiantes expresen «bastante interés», pero no «mucho» o «máximos, ni siquiera en los niveles de COU, en donde las opciones son elegidas por ellos mismos.

Observando estas medias totales, la asignatura que más interés despierta es Lengua y Literatura, seguida muy de cerca por Ciencias Naturales. Estos datos corroboran investigaciones anteriores en relación con el interés para la vida diaria y cultura general, pero no para otros aspectos, como el interés para estudios y trabajos posteriores, el gusto, etc. (Afonso López, et al., 1991).

Si analizamos la figura $I$, podemos ver la distribución de porcentajes de los intervalos de puntuación que ha recibido cada asignatura.

Como vemos, si consideramos los valores centrales ( 3 y 4), Ciencias Naturales es la asignatura que más interés despierta, si bien no se dan diferencias significativas.

\section{A.2. Interés de las asignaturas por niveles}

Con objeto de particularizar la información, manejamos ahora dos variables: asignatura y nivel. Los resultados obtenidos figuran en la tabla $\mathrm{V}$.

Observando los datos obtenidos para $1^{\circ}$ de BUP, vemos que las Ciencias Naturales ocupan el primer lugar en el interés del alumnado, seguido de Lengua y Literatura, Ciencias Sociales y Matemáticas, que reciben la puntuación más baja.

Si nos fijamos en COU-Ciencias, observamos que Ciencias Naturales (Biología y Geología) vuelve a ocupar el primer puesto del interés de los estudiantes, seguido de Lengua y Literatura, Matemáticas y Ciencias Sociales, aunque, como se ve, Ias diferencias son bastante pequeñas.

Centrando la atención en COU-Letras, observamos que la asignatura que más interés despierta es Lengua y Literatura, seguida por Matemáticas, Ciencias Naturales y Ciencias Sociales, siendo este último dato especialmente llamativo, si bien es cierto que no se dan diferencias significativas; no lo resulta tanto el hecho de que las matemáticas reciban una puntuación tan alta, en relacion con las demás, pues se trata de una asignatura de matemáticas enfocada hacia el campo sociohumanístico que suele ser bien recibida por el alumnado que la cursa.

Los datos expuestos corroboran la consideración social de Matemáticas y de Lengua y Literatura como disciplinas más importantes que el resto (Afonso López et al., 1991).

Se observan diferencias altamente significativas $(99,5 \%)$ entre COU-Ciencias y COU-Letras para Lengua y Literatura al hacer las comparaciones internas. Las comparaciones, curso a curso, en el orden expuesto para Lengua y Literatura muestran resultados de igualdad; sin 
Tabla V

Interés de las asignaturas por niveles.

\begin{tabular}{|c|c|c|c|c|}
\hline & Matemáticas & Lengua y Literatura & Ciencias Naturales & Ciencias Sociales \\
\hline $1^{\circ} \mathrm{DE} \mathrm{BUP}$ & $2,65(0=1)$ & $2,94(\sigma=0,93)$ & $2,96(\sigma=0,75)$ & $2,76(\sigma=0,78)$ \\
\hline COU-CIENCIAS & $2,80(\sigma=0,79)$ & $2,90(\sigma=0,71)$ & $2,94(\sigma=0,77)$ & $2,57(\sigma=1,05)$ \\
\hline COU-LETRAS & $3,00(\sigma=0,83)$ & $3,18(\sigma=0,82)$ & $2,98(\sigma=0,91)$ & $2,85(\sigma=0,75)$ \\
\hline
\end{tabular}

Negrita: Valores máximos.

embargo, para COU-Letras y COU-Ciencias se muestran diferencias significativas $(\mathrm{t}=-2,85 ; \mathrm{g} .1 .=238 ; \mu=$ 0,005 ) en relación con esta asignatura. Los resultados que se obtienen figuran en la tabla VI.

En relación con estos datos, llama la atención, como hemos comentado, la mayor aceptación en COU-Letras por Matemáticas; el resultado de $1^{\circ}$ de BUP, más bajo, no sorprende por cuanto es manifiesta la actitud negativa o pasiva y la faIta de interés que generalmente muestran los estudiantes en relación con las matemáticas al llegar a los centros de bachillerato.
Vuelve a ser curiosa, dentro de la igualdad, la media más alta para Ciencias Naturales de los estudiantes de COULetras. Esto podría hacer pensar que sería conveniente establecer en el currículo de los estudiantes de letras alguna asignatura relativa a las ciencias en general. Realmente, este alumnado sólo cursa asignaturas de corte clásico incluidas en el ámbito de conocimiento de las ciencias humanas y sociales, a excepción de Matemáticas, que, como hemos visto, aceptan de buen grado. Por el contrario, la «especialización» por llamarlo de alguna manera, de los estudiantes de ciencias es menor, en el sentido de que en su currículo siguen figurando

Distribución de los procentajes de las distintas asignaturas por intervalos.

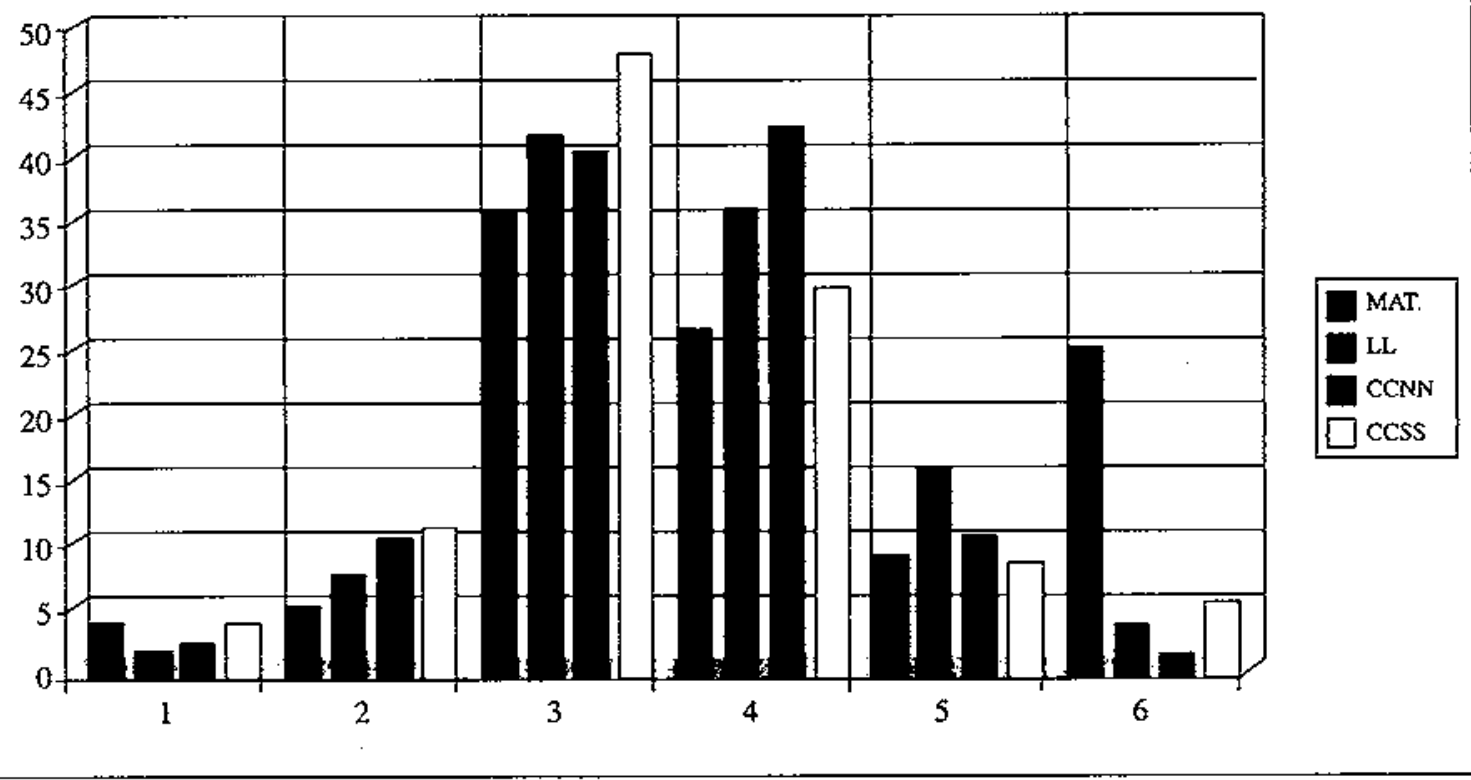


Tabla VI

Orden de los niveles por asignaturas y sus diferencias.

\begin{tabular}{|l|l|l|}
\hline MATEMÁTICAS & $\operatorname{COU}-\mathrm{L}(3,00)=\operatorname{COU}-\mathrm{C}(2,80)=1^{\circ} \mathrm{BUP}(2,65)$ & \\
\hline LENGUA Y LITERATURA & $\operatorname{COU}-\mathrm{L}(3,18)=1^{\circ} \mathrm{BUP}(2,94)=\operatorname{COU}-\mathrm{C}(2,90)$ & COU-LETRAS $>$ COU-CIENCIAS \\
\hline CIENCIAS NATURALES & $\operatorname{COU-L}(2,98)=1^{\circ} \mathrm{BUP}(2,96)=\operatorname{COU}-\mathrm{C}(2,94)$ & \\
\hline CIENCIAS SOCIALES & $\operatorname{COU} \cdot \mathrm{L}(2,85)=1^{\circ} \operatorname{BUP}(2,76)=\operatorname{COU}-\mathrm{C}(2,57)$ & \\
\hline
\end{tabular}

asignaturas de tipo lingiístico o humanístico que puntúan como las más bajas en el COU-Ciencias, pero ante las que tienen bastante interés.

Las Ciencias Sociales resultan mejor puntuadas por los estudiantes de COU Letras y peor por los de COU. Ciencias, como era de esperar.

Otro dato interesante que se desprende de la tabla VI es el hecho de que es COU-Letras el nivel que puntúa más alto en todas las asignaturas, el que demuestra tener intereses más altos en todas y cada una de ellas.

Sólo igualan o superan la puntuación $3 \mathrm{y}$, por lo tanto, muestran tener bastante interés, los estudiantes de COU. Letras para Matemáticas y para Lengua y Literatura, estando cercanos estos últimos a esa puntuación para Ciencias Naturales $(2,98)$; los de $1^{\circ}$ de BUP para Lengua y Literatura $(2,94)$; los de COU.Ciencias para Ciencias Naturales $(2,94)$, y este mismo nivel para Lengua y Literatura $(2,90)$.

\section{A.3. Interés de las asignaturas por centros}

Nos interesa ver ahora si existen diferencias, analizando la información en función de la asignatura y el centro como variables. El objetivo es estudiar la influencia que pueda ejercer la zona y el tipo de centro (urbano/rural) en los intereses generales del alumnado. Los datos obtenidos se muestran en la tabla VII.

Son, como se ve, muy significativamente diferentes los intereses del alumnado por Matemáticas de los centros IB-A con respecto a IB-B $(\mathrm{t}=-4,56 ; \mathrm{g} . \mathrm{l} .=170 ; \mu=0) \mathrm{e}$ IB-C $(\mathrm{t}=-3,23 ; \mathrm{g} .1 .=221 ; \mu=0,001)$. En Ciencias Naturales se observan diferencias muy significativas entre el $\mathrm{IB} \cdot \mathrm{A}$ y el IB $\cdot \mathrm{B}(\mathrm{t}=-3,69 ; \mathrm{g} . \mathrm{l} . \neq 242 ; \mu=0)$, así como entre éste y el IB-C $(\mathrm{t}=-3,14 ;$ g.l. $=257 ; \mu=$ 0,002 ).

Resulta especialmente llamativo que el IB-A ocupe en tres asignaturas (Matemáticas, Lengua y Literatura y Ciencias Naturales) la primera posición, habiendo mani. festado sus estudiantes un interés bastante alto; por el contrario, en Ciencias Sociales muestran el interés más bajo. Como se recordará, se trata de un centro comarcal grande de la zona norte de la isla de Tenerife, zona con una densidad de población elevada y más concentrada que en la zona sur, de la que el centro representativo es el IB-B. Estas diferencias podrían justificar que este último centro manifieste los intereses más bajos, excepto en Ciencias Sociales, asignatura en la que exponen el

Tabla VII

Interés de las asignaturas por centros.

\begin{tabular}{|c|c|c|c|c|}
\hline & Matemáticas & Lengua y Literatura & Ciencias Naturales & Ciencias Sociales \\
\hline IB-A & $3,13(\sigma=0,80)$ & $3,06(\sigma=0,73)$ & $3,07(\sigma=0,77)$ & $2,65(\sigma=0,90)$ \\
\hline IB-B & $2,53((\sigma=0,90)$ & $2,86(\sigma=0,90)$ & $2,69(\sigma=0,80)$ & $2,84(\sigma=0,86)$ \\
\hline IB-C & $2,77(\sigma=0,83)$ & $3,05(\sigma=0,87)$ & $3,01(\sigma=0,80)$ & $2,73(\sigma=0,85)$ \\
\hline
\end{tabular}

Negrita: Valores máximos en cada centro. 
Tabla VIII

Preferencias de asignaturas por centros.

\begin{tabular}{|c|c|c|c|c|}
\hline IB-A & MAT: $\mathbf{3 , 1 3}$ & CCNN: 3,07 & LL: 3,06 & CCSS: 2,65 \\
\hline IB-B & LL: 2,86 & CCSS: 2,84 & CCNN: 2,69 & MAT: 2,53 \\
\hline IB-C & LL: 3,05 & CCNN: 3,01 & MAT: 2,77 & CCSS: 2,73 \\
\hline
\end{tabular}

Negrita: Valores máximos.

más alto. El IB-C muestra en todos Ios casos una posición intermedia.

Si ordenamos, dentro de cada centro, las preferencias por asignaturas, obtenemos la tabla siguiente.

Siempre teniendo en cuenta que las diferencias son muy bajas, observamos que Ciencias Naturales ocupa una posición central (dos $2^{\circ}$ y un $3 r$. puesto); Lengua y Literatura muestra para los estudiantes un alto interés (dos $1^{\circ}$ y un $\left.3^{\circ}\right) ;$ Matemáticas y Ciencias Sociales muestran diferencias discrepantes entre los centros, siendo esta última la que menos puntuación recibe en dos de ellos y no llegando, tan siquiera, a «bastante interés».

Como se ve, en el IB-A la asignatura que más interés despierta es Matemáticas, y la que menos Ciencias Sociales. En el IB-C, to que más interesa es Lengua y Literatura y lo que menos, Ciencias Sociales. El IB-B muestra una tendencia diferente, interesándose más por Lengua y Literatura y menos por Matemáticas.

\section{A.4. Interés de los temas por asignaturas}

Para desarrollar este apartado, optamos por presentar los datos relativos a cada una de las asignaturas como subapartados. De este modo, podemos ver los valores totales por asignatura por centro y por nivel, por una parte, $\mathrm{y}$, por otra, los temas que más (señalados con negrita) y que menos (señalados con subrayado) interés despiertan en el alumnado; de igual manera, podemos observar las diferencias encontradas entre los máximos y los mínimos de las distintas asignaturas objeto de estudio.

\section{A.4.1. Matemáticas}

Tomando como referencia la asignatura, barajamos dos variables: centro y nivel. Los datos obtenidos de esta manera se reflejan en la tabla IX.

En $1^{\circ}$ de BUP no hay diferencias entre el IB-A y el IB-C, pero sí se dan diferencias muy significativas

Interés por Matematicas en centros y niveles.

\begin{tabular}{|l|c|c|c|}
\hline & IB-A & IB-B & IB-C \\
\hline $1^{\circ}$ DE BUP & $3,21(\sigma=0,78)$ & $\underline{2,23}(\sigma=1,05)$ & $2,93(\sigma=0,80)$ \\
\hline COU-CIENCIAS & $3,08(\sigma 0,86)$ & $2,83(\sigma=0,66)$ & $2,60(\sigma=0,71)$ \\
\hline COU-LETRAS & $3,15(\sigma=0,78)$ & $2,85(\sigma=0,55)$ & $2,90(\sigma=1)$ \\
\hline
\end{tabular}

Negrita: Vaior máximo.

Subrayado: Valor mínimo. 
Tabla X

Interés de los distintos temas de Matemáticas por niveles.

\begin{tabular}{|l|c|c|c|c|}
\hline Temas de Matemáticas & $1^{\circ} \mathrm{BUP}$ & COU-C & COU-L & $\tilde{X}$ \\
\hline COMBINATORIA Y PROBABILIDAD & $\underline{1,65}$ & $\underline{1,91}$ & 2,67 & $\underline{2,10}$ \\
\hline ESTADISTICA & 2,31 & 1,93 & $\mathbf{3 , 4 9}$ & 2,55 \\
\hline FUNCIONES Y GRÁFICAS & 3,16 & 3,05 & 2,87 & 3,04 \\
\hline GEOMETRÍA & 2,54 & 2,73 & $\underline{2,10}$ & 2,49 \\
\hline NÚMEROS Y OPERACIONES & 3,54 & $\mathbf{3 , 7 4}$ & 3,47 & $\mathbf{3 , 5 9}$ \\
\hline SISTEMAS DE ECUACIONES & $\mathbf{3 , 6 2}$ & 3,55 & 3,41 & 3,54 \\
\hline
\end{tabular}

Negrita: Valores máximos.

Subrayado: Valores mínimos.

$(99,7 \%)$ entre cada uno de éstos y el IB-B $(t=-3,08$; g.I. $=48 ; \mu=0,003)(t=-3,02 ; \mathrm{g} \cdot \mathrm{l}=65 ; \mu=0,004)$. En COUCiencias, las diferencias encontradas entre el IB-A y el IB-C son altamente significativas $(99,5 \%)(\mathrm{t}=-3$; g. $\mathrm{I}$. $=$ $96 ; \mu=0,003$ ).

Los valores medios obtenidos para Matemáticas oscilan entre 2,23 (mínimo) y 3,21 (máximo), ambos presentados por el alumnado de $1^{\circ}$ de BUP. Este valor se puede interpretar como un interés suficiente, aunque, si lo comparamos con Ciencias Sociales, es mayor y, si comparamos con Lengua y Literatura y con Ciencias Naturales, es menor.

$\mathrm{El}$ interés mayor, en relación con los temas seleccionados para el cuestionario, to demuestran los estudiantes de $1^{\circ}$ de BUP del IB-A; el menor, con diferencias significativas, lo manifiestan los del mismo nivel del IB-B.

Analicemos ahora las medias relativas a cada uno de los temas elegidos, por niveles y el valor medio (Tabla X).

Los valores medios para Matemáticas oscilan entre 2,10 (Combinatoria y Probabilidad) y 3,59 (Números y Operaciones). Si atendemos a estos valores medios, el orden de preferencia en los temas que se han presentado al alumnado sería el siguiente: Números y Operaciones, Sistemas de Ecuaciones, Funciones y Gráficas, Estadística, Geometría, y Combinatoria y Probabilidad.

Analizando Ios datos por niveles, se observa que los estudiantes de $1^{\circ}$ de BUP muestran el máximo interés por Sistemas de Ecuaciones $(3,62)$, y el mínimo por Combinatoria y Probabilidad $(1,65)$. El primero de ellos es un interés más cercano a «mucho» que a «bastante», mientras que el segundo es cercano a "poco interés».
Para COU-Ciencias, el tema que más interés despierta es Números y Operaciones $(3,74)$, cercano a «mucho interés»; el tema menos considerado es Combinatoria y Probabilidad $(1,91)$, al igual que en $1^{\circ}$ de BUP, lo que representa solamente "poco interés».

El alumnado de COU-Letras muestra «bastante interés» por la Estadística $(3,49)$, como era de esperar. Su menor interés lo despierta la Geometría $(2,10)$.

Los intereses manifestados para los distintos temas seleccionados presentan algunas diferencias en función del centro. Así, por ejemplo, el primer tema, Combinatoria y Probabilidad, muestra una diferencia muy significativa entre el IB-B y el IB-C $(t=-4,4 \mathrm{l}$; g.l. $=65$; $\mu=0$ ) en el nivel de $1^{\circ}$ de BUP. Son significativamente diferentes los resultados obtenidos para este tema y nivel entre los centros IB-A y el $\mathrm{IB}-\mathrm{B}(\mathrm{t}=-3,41 ; \mathrm{g} .1 \mathrm{l}=49$; $\mu=0,001$ ). La diferencia entre el IB-A y el IB-C en eI nivel COU-Ciencias para este tema es muy significativa $(t=-4,06 ; g . I=I 01 ; \mu=0)$.

\section{A.4.2. Lengua y Literatura}

Observemos en primer lugar los datos totales de esta asignatura por nivel y centro (Tabla XI).

Los valores medios que se han obtenido varían entre 2,69 y 3,41 . Considerando una escala de 0 a 5 como la que se proponía, estos datos pueden suponer un interés suficiente, mayor, por ejemplo, que el que se muestra para Ciencias Sociales.

El alumnado de COU-Letras del IB-A es el que explicita un mayor interés por esta asignatura, en función de los temas del cuestionario. Los estudiantes del COU-Cien- 
Tabla XI

Interés por Lengua y Literatura en centros y niveles.

\begin{tabular}{|l|c|c|c|}
\hline & IB-A & IB-B & IB-C \\
\hline $1^{\circ}$ DE BUP & $3,04(\sigma=0,81)$ & $2,75(\sigma=1,07)$ & $3,05(\sigma=0,90)$ \\
\hline COU-CIENCIAS & $2,99(\sigma=0,65)$ & $3,01(\sigma=0,62)$ & $\underline{2,69}(\sigma=0,81)$ \\
\hline COU-LETRAS & $3,41(\sigma=0,71)$ & $2,92(\sigma=0,80)$ & $3,27(\sigma=0,83)$ \\
\hline
\end{tabular}

Negrita: Valor máximo.

Subrayado: Valor mínimo.

cias deI IB-C son los que valoran de peor manera Lengua y Literatura.

Observemos, a continuación, los resultados obtenidos para cada uno de los temas; los datos se presentan en la tabla XII.

La dispersión de medias entre los distintos temas de Lengua y Literatura seleccionados para el cuestionario varía entre 2,64 (Escenificación) y 3,87 (Lecturas). Teniendo en cuenta los valores medios, el orden de preferencia del alumnado por los temas que se han presentado, sería el siguiente: Lectura, Expresión Oral, Creación Literaria, Análisis Morfosintáctico, Análisis de Textos, y, por último, Escenificación.
Para todos los estudiantes, el mayor interés, lo tiene la Lectura $(3,89,3,81$ y 3,92 respectivamente); como se ve, son valores muy cercanos a «mucho interés». El segundo Iugar lo muestra Expresión Oral $(3,20,3,28$ y 3,26 respectivamente).

Los datos expuestos resultan especialmente Ilamativos, dado que, en general, nuestro alumnado adolece de una expresión correcta, tanto oral como escrita y confiesa una muy baja afición por la lectura. De hecho, es en ésta última donde, básicamente, estaría la solución al primero de los problemas planteados en este terreno. El profesorado constata además las grandes dificultades que tiene que sortear para hacer que los estudiantes, incluso de modo obligatorio, lean algunos artículos o libros a lo

Tábla XII

Interés de los distintos temas de Lengua y Literatura por niveles.

\begin{tabular}{|c|c|c|c|c|}
\hline Temas de Lengua y Literatura & $1^{\circ} \mathrm{BUP}$ & COU-C & COU-L & $\bar{X}$ \\
\hline ANÁLISIS DE TEXTOS & $\underline{2,44}$ & $\underline{2.48}$ & 3,18 & 2,68 \\
\hline ANÁLISIS MORFOSINTÁCTICO & 2,57 & 2,73 & 2,90 & 2,72 \\
\hline CREACION LITERARIA & 2,84 & 2,65 & 3,22 & 2,89 \\
\hline ESCENIFICACIÓN & 2.75 & 2,56 & $\underline{2,60}$ & 2.64 \\
\hline EXPRESION ORAL & 3,20 & 3,28 & 3,26 & 3,25 \\
\hline LECTURAS & 3,89 & 3,81 & 3,92 & 3,87 \\
\hline
\end{tabular}

Negrita: Valores máximos.

Subrayado: Valores mínimos. 


\begin{tabular}{|l|c|c|c|}
\hline & IB-A & IB-B & IB-C \\
\hline I $^{\alpha}$ DE BUP & $3,22(\sigma=0,69)$ & $\underline{2,59}(\sigma=0,72)$ & $3,02(\sigma=0,68)$ \\
\hline COU-CIENCIAS & $2,87(\sigma=0,82)$ & $2,72(\sigma=0,44)$ & $3,06(\sigma=0,78)$ \\
\hline COU-LETRAS & $3,20(\sigma=0,77)$ & $2,82(\sigma=1,04)$ & $2,97(\sigma=0,89)$ \\
\hline
\end{tabular}

Negrita: Valor máximo.

Subrayado: Valor mítimo.

largo del curso, lo que se produce, de modo general, con grandes protestas y reticencias.

No se han detectado diferencias significativas para el primero, el cuarto y el sexto temas si analizamos los diferentes centros. Entre el IB-A y el IB-B, para el segundo tema (Análisis Morfosintáctico), en el nivel de $1^{\circ}$ de BUP se ha observado una diferencia muy significativa $(\mathrm{t}=3,71 ; \mathrm{g} .1$. $=110 ; \mu=0)$. También son muy significativas las diferencias entre estos centros, para este mismo nivel, en el tema tercero (Creación Literaria) $(\mathrm{t}=-3,51 ; \mathrm{g} .1 .=109 ; \mu=0,001)$. Las diferencias para el quinto tema, en este nivel y con los mismos centros, son sólo significativas $(\mathrm{t}=-2,73 ; \mathrm{g} .1 .=11 \mathrm{I} ; \mu=0,007)$. Para el mismo tema, estos centros son muy significativamen- te diferentes en COU-Letras $(t=-3,39 ;$ g.l. $=51 ; \mu=$ 0,001 ).

\section{A.4.3. Ciencias Naturales}

Los datos obtenidos para esta asignatura, usando como variables el centro y el nivel, se muestran en la tabla XIII.

Los estudiantes de $1^{\circ}$ de BUP del IB-A son los que muestran mayor interés por Ciencias Naturales, con respecto a los temas del cuestionario. El menor interés lo manifiesta el alumnado de $1^{\circ}$ de BUP del IB-B. Las diferencias entre el IB-A y el IB-B son muy significativas $(99,9 \%)(t=-4,59 ;$ g.l. $=105 ; \mu=0)$.

Tabla XIV

Interés por los distintos temas de Ciencias Naturales en los diferentes niveles.

\begin{tabular}{|l|c|c|c|c|}
\hline Temas de Ciencias Naturales & $1^{\circ}$ BUP & COU-C & COU-L & $\bar{X}$ \\
\hline AGENTES GEOLOGICOS EXTERNOS & 2,82 & 2,83 & 2,53 & 2,74 \\
\hline ECOLOGfA & 3,67 & 3,56 & 3,80 & 3,67 \\
\hline LA CÉLULA Y SU FUNCIONAMIENTO & 2,72 & 2,97 & 2,59 & 2,77 \\
\hline MINERALES Y ROCAS & 2,30 & $\underline{1,84}$ & $\underline{2,50}$ & $\underline{2,19}$ \\
\hline SERES VIVOS & 4,17 & $\mathbf{3 , 8 7}$ & $\mathbf{3 , 9 4}$ & 4 \\
\hline TECTÓNICA DE PLACAS & $\underline{2,07}$ & 2,56 & 2,51 & 2,38 \\
\hline
\end{tabular}

Negrita: Valores máximos.

Subrayado: Valores minimos. 
Los valores medios obtenidos oscilan entre 2,59 y 3,86 , lo que se podría interpretar como un interés suficiente $o$ bueno, si tenemos en cuenta que la escala varía entre 0 y 5 .

En el IB-A la puntuación más alta se obtiene en el alumnado de $1^{\circ}$ de BUP y desciende en ambos COU. En el IB-B la puntuación más baja se presenta en $1^{\circ}$ de BUP, aumentando en ambos $\mathrm{COU}$, más en Letras que en Ciencias. El lB-C muestra la mayor puntuación en COUCiencias, seguida con muy poca diferencia por $1^{\circ} \mathrm{de}$ BUP y por COU-Letras. Estos datos no se ajustan claramente, como se ve, a los resultados obtenidos por Yager y Penick (1984), según los cuales, a medida que se cursan más años, el gusto por las ciencias va disminuyendo, si tomamos los resultados como indicadores del interés a lo largo de la escolaridad. Tampoco parece que se mantenga el interés por la ciencia con los años de estudio, como apuntaba Serrano (1988), pues se producen aumentos y disminuciones según los centros y opciones.

Los resultados relativos a cada uno de los temas reflejan una diversidad grande en relación con los intereses del alumnado; se presentan en la tabla XIV.

Los valores medios para los temas de Ciencias Naturales oscilan entre 2,19 (Minerales y Rocas) y 4 (Seres Vivos). Utilizando los valores medios como criterio, nos quedaría el orden de temas siguiente: Seres Vivos, Ecología, La Célula y su funcionamiento, Agentes Geologicos Externos, Tectónica de Placas y, por último, Minerales y Rocas. Obsérvese que son los temas de Geología los que reciben menor puntuación $(2,74 / 2,38 / 2,19$ respectivamente). Teniendo en cuenta que los docentes de Ciencias Naturales en su mayoría son biólogos, un conocimiento menos profundo de la disciplina de la Geología puede influir en el hecho de que se utilicen menos recursos y se llegue menos al alumnado.
El orden establecido se mantiene tanto para COU-Ciencias como para COU-Letras; $1^{\circ}$ de BUP mantiene los dos primeros temas, pero prefiere los Agentes Geológicos Externos a la Célula, y Minerales y Rocas a Tectónica de Placas.

Si tenemos en cuenta las variaciones de los valores de estos temas por centros, no hay diferencias en los temas primero, cuarto y sexto. Para el segundo tema, son muy significativas $(99,9 \%)(\mathrm{t}=-3,84 ; \mathrm{g} .1 .=105 ; \mu=0)$ las diferencias entre eI IB-A y el IB-B en $1^{\circ}$ de BUP, y significativas $(t=-2,78 ; g .1 .=77 ; \mu=0,007$ ) entre el IB-B y el IB-C, para el mismo tema en dicho nivel. En el tema tercero, las diferencias detectadas son altamente significativas $(99,5 \%)(\mathrm{t}=-2,99$; g.I. $=90$; $\mu=0,004)$ entre el IB-A y el $\mathrm{B}$ B-C, para $1^{\circ}$ de BUP; COU-Ciencias muestra, para este mismo tema, diferencias que son muy significativas $(99,9 \%)(\mathrm{t}=-4,47 ; \mathrm{g}, \mathrm{l}=$ $78 ; \mu=0$ ) entre el IB-A y el IB-B y entre éste y el IB-C $(t=-5,83 ; g . l=84 ; \mu=0)$. COU-Letras muestra una diferencia significativa $(99,1 \%)$ entre el IB-A y el IB-B $(t=-2,75 ; g .1 .=55 ; \mu=0,008)$. En el quinto tema se vuelven a mostrar diferencias muy significativas entre los distintos centros en COU-Ciencias y entre el IB-A y el IB-B para $1^{\circ}$ de BUP $(t=-3,55$; g. $1 .=107$; $\mu=0,001$ ).

\section{A.4.4. Ciencias Sociales}

Observemos los datos globales por centro y nivel; éstos son Ios que se muestran en la tabla XV.

Tomando como referencia los temas expuestos en el cuestionario, son los estudiantes de $1^{\circ}$ de BUP del IB-B los que muestran un interés más alto. El alumnado de COU-Ciencias del IB-A es el que manifiesta un menor interés.

\begin{tabular}{|l|c|c|c|}
\hline & IB-A & IB-B & IB-C \\
\hline $1^{\circ}$ DE BUP & $2,59(\sigma=0,76)$ & $2,96(\sigma=0,86)$ & $2,71(\sigma=0,51)$ \\
\hline COU-CIENCIAS & $2,49(\sigma=0,97)$ & $2,82(\sigma=1,16)$ & $2,54(\sigma=1,08)$ \\
\hline COU-LETRAS & $2,95(\sigma=0,89)$ & $2,61(\sigma=0,42)$ & $2,91(\sigma=0,73)$ \\
\hline
\end{tabular}

Negrita: Valor máximo.

Subrayado: Valor minimo. 
Tabla XVI

Interés por los distintos temas de Ciencias Sociales por niveles.

\begin{tabular}{|c|c|c|c|c|}
\hline Temas de Ciencias Sociales & $1^{\circ} \mathrm{BUP}$ & $\mathrm{COU}-\mathrm{C}$ & $\cos -\mathrm{L}$ & $\bar{x}$ \\
\hline CAPITALISMO Y SOCIALISMO & 2,17 & 2,88 & 3,45 & 2,79 \\
\hline EDAD MEDIA & 2,89 & 2,65 & 2,60 & 2,72 \\
\hline EL RENACIMIENTO & 3,32 & 2,31 & 2,90 & 2,85 \\
\hline REVOLUCIONES & 3,64 & 2,97 & 3,30 & 3,22 \\
\hline SECTORES ECONÓMICOS & 2,62 & 2,94 & 2,96 & 2,83 \\
\hline SISTEMAS SOCIOPOLITICOS GRIEGOS & $\underline{2.00}$ & 1,67 & 1.87 & 1,85 \\
\hline
\end{tabular}

Negrita: Valores máximos.

Subrayado: Valotes mínimos.

Los valores medios obtenidos, como se ve, oscilan entre 2,49 y 2,96, lo que se puede considerar como un interés suficiente, si bien, comparándolo con las otras tres asignaturas, es menor.

Analicemos ahora los resultados por temas, como se expresa en la tabla XVI.

Las oscilaciones que muestran las medias, según los temas, varían entre 1,85 (Sistemas Sociopolíticos Griegos) y 3,22 (Revoluciones Francesa, Industrial, Burguesa). Si usamos estos valores medios para ordenar los distintos contenidos, según el interés expresado por el alumnado, quedaría lo siguiente: Revoluciones (Francesa, Industrial, Burguesa), el Renacimiento, Sectores Económicos, Capitalismo y Socialismo, Edad Media y, en último lugar, los Sistemas Sociopolíticos Griegos. Este orden sólo es válido para valores medios, pues los tres niveles manifiestan tendencias discrepantes, coincidiendo solamente en el tema de menor interés. Se observa en esta asignatura, en relación con las anteriores, una distribución más dispersa.

No se han detectado diferencias si se analizan Ios valores referidos a tos tres centros, con la excepción del sexto tema en $1^{\circ}$ de BUP entre el IB-A y el IB-B, que muestran una diferencia altamente significativa $(99,5 \%)(t=2,88$; g.l. $=101 ; \mu=0,005$ ).

\section{A.5. Valoración conjunta del interés por las cuatro asignaturas}

Conviene ahora, sobre todo, a juzgar por los últimos datos presentados, hacer un análisis de las diferencias entre máximos y mínimos en los diferentes temas selec-

Tabla XVII

Valores máximos y minimos y diferencias en la puntuación de distintos temas en las diferentes asignaturas.

\begin{tabular}{|l|c|c|c|c|}
\hline & Matemáticas & $\begin{array}{c}\text { Lengua } \\
\text { y Literatura }\end{array}$ & $\begin{array}{c}\text { Ciencias } \\
\text { Naturales }\end{array}$ & $\begin{array}{c}\text { Ciencias } \\
\text { Sociales }\end{array}$ \\
\hline MÁXIMO & 3,59 & 3,87 & $\mathbf{4 , 0 0}$ & 3,22 \\
\hline MÍNIMO & 2,10 & 2,64 & 2,19 & $\underline{1,85}$ \\
\hline DIFERENCIA & 1,49 & 1,23 & $\mathbf{1 , 8 1}$ & 1,37 \\
\hline
\end{tabular}

Negrita: Valores máximos.

Subrayado: Valores mínimos. 
Tabla XVIII

Puntuaciones y números de orden de los distintos temas en las diferentes asignaturas.

\begin{tabular}{|c|c|c|c|}
\hline Matemáticas & Lengua y Literatura & Ciencias Naturales & Ciencias Sociales \\
\hline $2,10(\mathbf{2 2})$ & $2,68(16)$ & $2,74(14)$ & $2,79(12)$ \\
\hline $2,55(\mathbf{1 8})$ & $2,72(15)$ & $3,67(2)$ & $2,72(15)$ \\
\hline $3,04(8)$ & $2,89(9)$ & $2,77(13)$ & $2,85(10)$ \\
\hline $2,49(19)$ & $2,64(17)$ & $2,19(21)$ & $3,22(7)$ \\
\hline $3,59(4)$ & $3,25(6)$ & $4(1)$ & $2,83(11)$ \\
\hline $3,54(5)$ & $3,87(3)$ & $2,38(20)$ & $1,85(23)$ \\
\hline
\end{tabular}

cionados para cada una de las asignaturas; estas comparaciones figuran en la tabla XVII.

Como puede verse, la mayor dispersión la presenta Ciencias Naturales, mostrando, además, la puntuación más alta de todas las obtenidas (Seres Vivos). La menor dispersión se observa en Lengua y Literatura, lo que quiere decir que los diferentes temas despiertan intereses más similares que en las otras asignaturas. Esto puede explicar que la media sea algo superior a Ia de Ciencias Naturales. El tema menos valorado, incluso con diferencia, es Sistemas Sociopolíticos Griegos; si añadimos a esto que en Ciencias Sociales el máximo también es menor, se justifica la media más baja de esta asignatura, en relación con las demás, y siempre teniendo en cuenta la selección de temas que se ha hecho.

Si ordenamos Ios temas de todas las asignaturas de mayor a menor puntuación, obtenemos Ia tabla siguiente (Tabla XVIII); en ella hemos puesto el número de orden al lado de la puntuación recibida.

Situando en cada asignatura los puestos recibidos en la relación total de los temas encuestados, se obtienen los resultados expuestos en Ia tabla XIX.
Como puede observarse, Ciencias Naturales presenta los temas mejor puntuados (posiciones $1^{a}$ y $2^{a}$ ), quedando el resto más repartido: Matemáticas $\left(4^{\circ}, 5^{\circ}\right.$ y $8^{\circ}$ lugar); Lengua y Literatura $\left(3^{\circ}, 6^{\circ}\right.$ y $9^{\circ}$ puesto); Ciencias Sociales $\left(7^{\mathrm{a}}\right.$ y $10^{\mathrm{a}}$ posiciones). Se han utilizado los diez primeros como referencia.

De estos resultados no se desprende, en todo caso, que Ciencias Naturales sea la asignatura que ostenta el mayor interés del alumnado; más bien al contrario, Lengua y Literatura, y Matemáticas parecen resultar más interesantes. De lo anterior parece derivarse que no es el contenido seleccionado lo que justifica un mayor interés por Ciencias Naturales.

\section{B. La metodología desde el punto de vista del alum- nado}

Como se recordará, esta valoración se corresponde con la segunda parte del cuestionario; su finalidad es contrastar la segunda de las hipótesis formuladas, relativa a las diferencias posibles entre los docentes de las cuatro asignaturas en la forma de contemplar los distintos elementos de la metodología. Esta parte de la encuesta se añade como anexo $\mathrm{I}$. Hemos organizado los datos, para

Tabla XIX

Posiciones de los distintos temas en las diferentes asignaturas.

\begin{tabular}{|l|c|c|c|c|c|c|}
\hline MATEMÁTICAS & $4^{\circ}$ & $5^{\circ}$ & $8^{\circ}$ & $18^{\circ}$ & $19^{\circ}$ & $22^{\circ}$ \\
\hline LENGUA Y LITERATURA & $3^{\circ}$ & $6^{\circ}$ & $9^{\circ}$ & $15^{\circ}$ & $16^{\circ}$ & $17^{\circ}$ \\
\hline CIENCIAS NATURALES & $1^{\circ}$ & $2^{\circ}$ & $13^{\circ}$ & $14^{\circ}$ & $20^{\circ}$ & $21^{\circ}$ \\
\hline CIENCIAS SOCIALES & $7^{\circ}$ & $10^{\circ}$ & $11^{\circ}$ & $12^{\circ}$ & $15^{\circ}$ & $23^{\circ}$ \\
\hline
\end{tabular}


Tabla XX

Valores de los distintos ítems por asignatura, valor medio y media de cada factor.

\begin{tabular}{|c|c|c|c|c|c|c|}
\hline Ítem & Matemáticas & Lengua y Literatura & Ciencias Naturales & Ciencias Sociales & $\bar{x}$ & $\bar{X}_{\text {Factor }}$ \\
\hline 7 & $1.83(\sigma=0,75)$ & $2,08(\sigma=0,80)$ & $1,87(\sigma=0,83)$ & $1,91(\sigma=0,81)$ & 1,92 & 2,13 \\
\hline 12 & $2,45(\sigma=0,83)$ & $\underline{2.22}(\sigma=0,89)$ & $2,31(\sigma=0,79)$ & $2,39(\sigma=0,79)$ & 2,34 & $(\sigma=0,56)$ \\
\hline 10 & $0,64(\sigma=0,81)$ & $1,20(\sigma=0,90)$ & $0,86(\sigma=0,89)$ & $1,13(\sigma=0,86)$ & 0,95 & \multirow{3}{*}{$\begin{array}{c}1,22 \\
(\sigma=0,60)\end{array}$} \\
\hline 15 & $2,00(\sigma=1,01)$ & $1,79(\sigma=0,89)$ & $1,39(\sigma=0,94)$ & $\underline{1,33}(\sigma=0,88)$ & 1,63 & \\
\hline 17 & $1,10(\sigma=0,94)$ & $\underline{1.04}(\sigma=0,87)$ & $1,07(\sigma=0,89)$ & $1,11(\sigma=0,88)$ & 1,08 & \\
\hline $1 \mathrm{I}$ & $1.17(\sigma=0,98)$ & $1,46(\sigma=0,93)$ & $1,25(\sigma=0,96)$ & $1,33(\sigma=0,93)$ & 1,30 & \multirow{3}{*}{$\begin{array}{c}1,45 \\
(\sigma=0,48)\end{array}$} \\
\hline 16 & $1.69(\sigma=0,90)$ & $2,03(\sigma=0,78)$ & $1,79(\sigma=0,84)$ & $1,81(\sigma=0,80)$ & 1,83 & \\
\hline 20 & $1,21(\sigma=1,04)$ & $1.07(\sigma=0,99)$ & $1,31(\sigma=1,06)$ & $1,22(\sigma=1,03)$ & 1,20 & \\
\hline 9 & $1,96(\sigma=0,90)$ & $2,32(\sigma=0,82)$ & $2,43(\sigma=0,82)$ & $2,56(\sigma=0,63)$ & 2,32 & \multirow{3}{*}{$\begin{array}{c}1,73 \\
(\sigma=0,61)\end{array}$} \\
\hline 14 & $0,51(\sigma=0,83)$ & $1,12(\sigma=0,95)$ & $1,64(\sigma=1,03)$ & $1,46(\sigma=0,99)$ & 1,88 & \\
\hline 19 & $1,71(\sigma=1,08)$ & $1,45(\sigma=1,06)$ & $1,82(\sigma=1,03)$ & $1,78(\sigma=1,00)$ & 1,69 & \\
\hline 8 & $0,40(\sigma=0,71)$ & $0,95(0=0,93)$ & $1,22(\sigma=1,02)$ & $0,89(\sigma=0,86)$ & 0,87 & \multirow{3}{*}{$\begin{array}{c}1,65 \\
(\sigma=0,59)\end{array}$} \\
\hline 13 & $2.06(\sigma=0,98)$ & $2,18(\sigma=0,86)$ & $2,18(\sigma=0,91)$ & $2,12(\sigma=0,91)$ & 2,14 & \\
\hline 18 & $1.84(\sigma=1,25)$ & $1,91(\sigma=1,17)$ & $2,19(\sigma=1,10)$ & $1,89(\sigma=1,22)$ & 1,96 & \\
\hline
\end{tabular}

Negrita: Valores máximos.

Subrayado: Valores mínimos.

conseguir, por una parte, una mayor claridad, en torno a los valores globales y, por otra, a los valores pormenorizados por elementos, utilizando como variables la asignatura, y asignatura y nivel. La escala utilizada para obtener la información relativa a esta cuestión, como ya se ha expuesto, tiene cuatro niveles $(0,1,2,3)$.

\section{B.1. Valores totales}

Analizando los datos globales por asignaturas, se obtienen los resultados que figuran en la tabla XX.

A juzgar por estos resultados, parece ser que Ciencias Naturales es la asignatura en la que se dan los planteamientos más tradicionales.

Si nos centramos en las estrategias (ítems 7 y 12), las diferencias entre las cuatro asignaturas son muy bajas, oscilando en un margen de 0,25 puntos para el primer ítem y de 0,23 para el segundo. Los datos muestran que, para el alumnado, Ios docentes seleccionamos materiales y actividades en una medida aceptable que sólo llega a «bastante» para Lengua y Literatura, y que tiene su menor valor en Matemáticas. Este interés que parecen captar los estudiantes por parte del profesorado, en relación con los materiales que usa, contrasta con la alta puntuación presentada en relación con el protagonismo del mismo. Puede observarse que en las cuatro asignaturas es superior al 2, incluso bastante, en algunas; la asignatura que advierte un menor protagonismo de sus docentes, dentro de lo que cabe, es Lengua y Literatura.

Fijándolos en los ítems relativos a actividades y tareas $(10,15$ y 17$)$, los debates y discusiones que se mantienen en las clases son muy pocos, en general, siendo estas actividades significativamente poco frecuentes en Matemáticas y en Ciencias Naturales; la asignatura en la que más se desarrollan es Lengua y Literatura, pero, como se ve, «poco» o "casi nunca». 
El tiempo de la clase se utiliza para hacer actividades, con una frecuencia bastante alta en Matemáticas; es de suponer que el alumnado haya interpretado como actividades las tareas de corrección de problemas. Tambiên es relativamente alta la proporción de tiempo que se ocupa con estos fines en Lengua y Literatura. En Ciencias Naturales y Ciencias Sociales este tipo de actividades o tareas ocupa «poco» tiempo dentro de las horas de clase.

Es poco frecuente desarrollar actividades de investigación para el aprendizaje en las cuatro asignaturas; Ciencias Sociales muestra el resultado más alto, pero Ia diferencia entre este valor y el mínimo (Lengua y Literatura) es solamente de 0,07 puntos.

La información relativa al papel del profesorado se ha obtenido con los ítems 11,16 y 20 . En líneas generales, los estudiantes consideran a los docentes poco flexibles y poco dispuestos a ofrecer distintas opciones en el trabajo de aula. Si bien las diferencias son pocas, la asignatura en la que se advierte menor flexibilidad es Matemáticas, siendo Lengua y Literatura la más dispuesta a mostrar distintas posibilidades y más flexible en relación con su profesorado. Es el profesorado de Lengua y Literatura el que consigue mantener un clima más apropiado para el trabajo; los docentes de Matemáticas, según los jóvenes, generan el clima menos adecuado.

Siguiendo la tendencia expuesta en los ítems anteriores, el profesorado de Lengua y Literatura es el más cercano al alumnado, siendo los docentes de Ciencias Naturales los más distantes. En todo caso, obsérvese que el valor máximo es de 1,31 , lo que significa que en líneas generales el profesorado es poco distante con el alumnado.
Los valores obtenidos en lo relativo a medios y recursos utilizados (ítems 9,14 y 19 ; libro de texto y explicaciones del docente) son muy altos, significando esto que habitualmente no se utilizan otros materiales. Ciencias Sociales presenta el valor más alto y Lengua y Literatura, el más bajo.

Lo anterior se ve reforzado por los datos obtenidos para el îtem 14. Prácticamente no se hacen actividades diversas en clase, no se utilizan distintos materiales en Matemáticas, siendo Ciencias Naturales la asignatura que presenta un mayor valor a este respecto, valor que tampoco llega a ser considerado «bastante».

En términos generales es cercana la consideración a «bastante» en relación con el comportamiento en clase y las pautas indicadas por el profesorado. Esto significa que son los docentes los que en una alta proporción determinan lo que se hace y lo que se usa en el aula.

De Ios resultados obtenidos para el entorno de aprendizaje (ítems 8,13 y 18 ), se desprende que el alumnado no se distribuye en grupos prácticamente nunca; solamente en Ciencias Naturales se hace este tipo de agrupamiento con poca frecuencia.

Los datos obtenidos en lo relativo al protagonismo del profesorado para distribuir el tiempo de clase revelan que dichas decisiones son dominio casi absoluto de los docentes en las cuatro asignaturas ya que en todas se supera el valor 2 . También son considerablemente altos los valores obtenidos en relación con la organización del espacio del aula de modo tradicional, pues se supera el 2 ( «bastante») en Ciencias Naturales para la distribución en filas del mobiliario, y se aproximan a esta cifra las otras tres asignaturas. Esto contrasta con el dato relativo

Tabla XXI

Valores medios por asignatura y nivel para estrategias.

\begin{tabular}{|c|c|c|c|c|c|}
\hline Item & Matemáticas & $\begin{array}{c}\text { Lengua } \\
\text { y Literatura }\end{array}$ & $\begin{array}{l}\text { Ciencias } \\
\text { Naturales }\end{array}$ & $\begin{array}{l}\text { Ciencias } \\
\text { Sociales }\end{array}$ & Nivel \\
\hline 7 & $2,1 \mathrm{I}$ & 2,10 & 1,89 & 2,16 & \multirow[t]{2}{*}{$1^{\circ} \mathrm{DE} \mathrm{BUP}$} \\
\hline 12 & 2,28 & 2,29 & 2,26 & 2,20 & \\
\hline 7 & 1.59 & 2,00 & 1,98 & 1,70 & \multirow[t]{2}{*}{ COU-C } \\
\hline I2 & 2,57 & 2,42 & 2,26 & 2,38 & \\
\hline 7 & $I, 81$ & 2,14 & 1,70 & 1,85 & \multirow[t]{2}{*}{ COU-L } \\
\hline 12 & 2,53 & $\$ .89$ & 2,44 & 2,61 & \\
\hline
\end{tabular}


Tabla XXII

Valores medios de las estrategias por asignatura y nivel.

\begin{tabular}{|l|c|c|c|c|}
\hline & $I^{\circ}$ de BUP & COU-Ciencias & COU-Letras & $\bar{X}$ \\
\hline MATEMÁTICAS & 2,20 & 2,07 & 2,17 & $\mathbf{2 , 1 5}$ \\
\hline LENGUA Y LITERATURA & 2,21 & 2,21 & $\underline{2,00}$ & $\mathbf{2 , 1 5}$ \\
\hline CIENCIAS NATURALES & 2,08 & 2,12 & 2,08 & $\underline{2,09}$ \\
\hline CIENCIAS SOCIALES & 2,18 & 2,04 & $\mathbf{2 , 2 3}$ & $\mathbf{2 , 1 5}$ \\
\hline
\end{tabular}

Negrita: Valores máximos.

Subrayado: Valores mínimos.

al ítem 8, según el cual Ciencias Naturales es la asignatura en la que más se trabaja en grupos, si bien su valoración es cercana a «poco».

\section{B.2. Valoración de la metodología por niveles}

Analizaremos estos datos en subapartados, utilizando como primer criterio los elementos de la metodología; en cada uno de ellos se desglosa la información por asignatura y nivel.

\section{B.2.1. Estrategias}

Si analizamos las medias por cursos, obtenemos la tabla que hay a continuación (Tabla XXI).

Es en Ciencias Sociales de $1^{\circ}$ de BUP donde el profesorado selecciona más materiales y actividades para el aprendizaje de sus estudiantes; en las Matemáticas de COU-Ciencias es donde menos se dedican los docentes a esta tarea. Esto contrasta con el protagonismo del profesorado de Ciencias Sociales, que es mayor en

Tabla XXIII

Valores medios de los distintos átems de actividades y tareas por asignatura y nivel.

\begin{tabular}{|c|c|c|c|c|c|}
\hline Item & Matemáticas & $\begin{array}{c}\text { Lengua } \\
\text { y Literatura }\end{array}$ & $\begin{array}{l}\text { Ciencias } \\
\text { Naturales }\end{array}$ & $\begin{array}{l}\text { Ciencias } \\
\text { Sociales }\end{array}$ & Nivel \\
\hline 10 & 0,86 & 1,17 & 0,84 & 1,22 & \multirow{3}{*}{$1^{\circ} \mathrm{DE}$ BUP } \\
\hline 15 & 2,13 & 1.88 & 1,40 & 1,65 & \\
\hline 17 & 1,24 & 1,02 & 1,03 & 1,20 & \\
\hline 10 & $\underline{0,43}$ & 1,01 & 0,91 & 1,19 & \multirow{3}{*}{ COU-C } \\
\hline 15 & 1,89 & 1,76 & 1,33 & 1,31 & \\
\hline 17 & 1,04 & 0.87 & 1,05 & 1,03 & \\
\hline 10 & 0,64 & 1,43 & 0,83 & 0,97 & \multirow{3}{*}{ COU-L } \\
\hline I5 & 1,98 & 1,72 & 1,47 & 0.97 & \\
\hline 17 & 0,99 & 1,25 & 1,13 & 1,08 & \\
\hline
\end{tabular}

Negrita: Valores máximos.

Subrayado: Valores mínimos. 
COU-Letras, siendo, el menor, el presentado por este mismo nivel en Lengua y Literatura.

El ítem 7 muestra diferencias altamente significativas $(99,6)(t=2,96 ;$ g. $1 .=240 ; \mu=0,003)$ entre $1^{\circ}$ de BUP y COU-Letras y muy significativas $\left(\mathrm{t}=5,08 ; \mathrm{g} . \mathrm{l}_{\mathrm{f}}=278\right.$; $\mu=0$ ) entre $1^{\circ}$ de BUP y COU-Ciencias, para Matemáticas. En esta misma asignatura, se observan diferencias altamente significativas $(99,6)$ entre los niveles de $1^{\circ}$ de BUP y COU-Ciencias para el ítem $12(t=-2,89$; g.l. = $279 ; \mu=0,004$ ).

En Lengua y Literatura no se observan diferencias significativas en el ítem 7 pero sí en el 12: tanto $1^{\circ}$ de BUP/ COU.Letras $(\mathrm{t}=3,49 ; \mathrm{g} .1 .=255 ; \mu=0,001)$ como COUCiencias/COU $\cdot$ Letras ( $\mathrm{t}=5,05 ; \mathrm{g}+\mathrm{h}_{\mathrm{f}} \neq 242 ; \mu=0$ ) son significativamente diferentes para el mismo.

Ciencias Naturales muestra diferencias altamente significativas $(99,5)$ para el f́tem 7 entre COU-Ciencias y COU-Letras $(t=2,81 ; g .1 .=263 ; \mu=0)$. No muestra diferencias en el f́tem 12 .

Ciencias Sociales presenta también discrepancias: $1^{\circ}$ de BUP es significativamente diferente tanto de COU. Ciencias como de COU-Letras $(99,9$ y 99,8 respectivamente) $(\mathrm{t}=4,69 ; \mathrm{g} . \mathrm{l} .=248 ; \mu=0)(\mathrm{t}=3, \mathrm{1} 2 ; \mathrm{g} . \mathrm{l}=235$; $\mu=0,002$ ) en el ítem 7 ; también lo son $1^{\circ}$ de BUP y COULetras para el ítem $12(t=-3,94 ; \mathrm{g} .1 . \neq 235 ; \mu=0)$.

Dada la naturaleza del alumnado y contrastando con el resto de los ítems, no se hace una enseñanza centrada en la investigación por parte del alumnado. Recuérdese que los centros seleccionados son de corte tradicional. Si hacemos una valoración conjunta de ambos ítems, obtenemos un valor $=2,13$, que viene a representar el tipo de estrategia usado por el profesorado, considerado globalmente. Este valor demuestra una clara tendencia hacia estrategias expositivas. Analicemos estos mismos valores por asignatura y nivel (Tabla XXII):
Como se ve, todos Ios valores superan el 2 , lo que, como decíamos anteriormente, representa un claro predominio de estrategias expositivas. Considerando los valores medios, la asignatura "menos expositiva» corresponde a Ciencias Naturales, estando las otras tres igualadas con una minima diferencia de 0,06 décimas.

El tipo de trabajo docente menos expositivo es el que se da por parte de los profesionales de Lengua y Literatura para el nivel de COU-Letras. El más expositivo vuelve a corresponder al mismo nivel para Ciencias Sociales.

$1^{\circ}$ de BUP y COU-Letras muestran una diferencia significativa para Lengua y Literatura $(99,3)(\mathrm{t}=2,74 ; \mathrm{g} . \mathrm{l}$. = $251 ; \mu=0,007)$. En esta misma asignatura ambos cursos de COU tienen diferencias altamente significativas $(99,5)$ $(\mathrm{t}=2,82 ; \mathrm{g} .1 .=237 ; \mu=0,005)$. Igualmente se muestran diferencias significativas $(99,1)(\mathrm{t}=-2,63 ; \mathrm{g} . \mathrm{l} .=223$; $\mu=0,009$ ) entre ambos COU en Ciencias Sociales.

\section{B.2.2. Actividades y tareas}

Los datos pormenorizados por asignatura y nivel para los ítems relativos a actividades y tareas figuran en la tabla siguiente (Tabla XXIII).

Ciencias Sociales de $1^{\circ}$ de BUP es la asignatura en la que más debates y discusiones se establecen, siendo en Matemáticas de COU-Ciencias donde menos se utilizan este tipo de actividades, llegando a ser casi nulo el resultado obtenido.

El valor más alto para las tareas realizadas en clase se asigna a Matemáticas de $1^{\circ}$ de BUP. Esto significa que en este nivel y asignatura el alumnado considera que la mayor parte de la clase se dedica «bastante» a hacer actividades, considerando probablemente dentro de las mismas la resolución tradicional o habitual de problemas. El valor más bajo en este aspecto lo presenta Ciencias Sociales de COU-Letras.

Tabla XXIV

Valores medios de actividades y tareas por asignatura y nivel.

\begin{tabular}{|c|c|c|c|c|}
\hline & $10^{\circ} \mathrm{de}$ BUP & COU-Ciencias & CoU-Letras & $\bar{X}$ \\
\hline MATEMATICAS & 1,40 & 1,12 & 1,20 & 1.24 \\
\hline LENGUA Y LITERATURA & 1,36 & 1,21 & 1,47 & 1,34 \\
\hline CIENCIAS NATURALES & 1,10 & 1,09 & 1,14 & 1,11 \\
\hline CIENCIAS SOCIALES & 1,35 & 1,18 & 1,02 & 1,19 \\
\hline
\end{tabular}


Lengua y Literatura de COU.Letras es la asignatura y nivel en el que, a juzgar por los resultados, se hacen más investigaciones, siendo el valor muy cercano, en todo caso, a «poco». Esta misma asignatura, pero en COUCiencias, presenta el valor más bajo. Esta situación resulta contradictoria, pues parece difícil aceptar que los docentes de este nivel en ambos COU utilicen tipos de actividades diferentes para lo mismo o con los mismos fines; en muchos casos son incluso los mismos profesores.

En la asignatura de Matemáticas, el ítem 10 muestra diferencias muy significativas $(99,9)(\mathrm{t}=4,60$; g.l. $=$ $281 ; \mu=0$ ) entre $1^{\circ}$ de BUP y COU-Ciencias. Los ítems 15 y 17 no presentan diferencias entre los distintos niveles.

Lengua y Literatura muestra diferencias muy significativas en los ítems 10 y 17 entre COU-Ciencias y COULetras $(\mathrm{t}=-3,63 ; \mathrm{g} . \mathrm{I} .=244 ; \mu=0)(\mathrm{t}=-3,53 ; \mathrm{g} . \mathrm{I}=242$; $\mu=0,001)$. No se observan diferencias en el ítem 15 .

Ciencias Naturales no presenta diferencias en ningún ítem entre los distintos niveles.

En Ciencias Sociales los ítems 10 y 17 no muestran diferencias entre niveles; las respuestas del ítem 15 son muy significativamente diferentes entre todos los niveles $\left(1^{\circ} \mathrm{de}\right.$ BUP/COU-Ciencias $(99,8)(t=3,19 ; \mathrm{g} \cdot 1 \mathrm{l}=245$; $\mu=0,002)$ y $1^{\circ}$ de BUP/COU-Letras $(-99,9)(t=6,12$; g.l. $=235 ; \mu=0)$; COU-Ciencias/COU-Letras $(99,8)$ $(\mathrm{t}=3,07 ; \mathrm{g} . \mathrm{h} .=224 ; \mu=0,002)$.
Valorando de modo conjunto los tres ítems se obtiene un valor de 1,22, que viene a tepresentar, dentro de la escala 0-3 que habíamos establecido, que en líneas generales no se utilizan actividades diversas; no se advierte por parte del alumnado un interés del profesorado por plantear alternativas diferentes en sus clases. Veamos este aspecto por asignatura y nivel (Tabla XXIV).

$1^{\circ}$ de BUP y COU-Ciencias son muy significativamente diferentes en relación con Matemáticas $(99,9)(\mathrm{t}=4,17$; g.l. $=278 ; \mu=0$ ); las diferencias entre $1^{\circ}$ de BUP y COULetras para esta asignatura son sólo significativas $(99,1)$ $(t=2,65 ; g . I .=240 ; \mu=0,009)$. Lengua y Literatura muestra diferencias muy significativas entre COU-Ciencias y COU-Letras $(99,9)(t=-3,40 ; g \cdot 1 .=241 ; \mu=0,001)$. En Ciencias Naturales no se han observado diferencias por niveles. Ciencias Sociales presenta diferencias muy significativas $(99,9)$ entre $1^{\circ}$ de BUP y COU-Letras $\langle t=$ 4,$26 ;$ g.1. $=230 ; \mu=0$ )

Los valores obtenidos muestran, como decíamos antes, una clara tendencia de los docentes de las diferentes asignaturas hacia la poca diversificación de las propuestas de actividades. Es especialmente significativa la posición que ocupan las asignaturas de Ciencias Naturales tanto en $1^{\circ}$ de BUP y COU-Ciencias como en los valores medios, sobre todo teniendo en cuenta la aceptación y el interés mostrado por los estudiantes por el contenido de dichas asignaturas. Esto puede interpretarse como un aprovechamiento escaso por parte de los docentes del potencial de los propios temas, lo que conduce a que, con una programación adecuada de las

Tabla XXV

Valores de los distintos ítems del papel del profesorado por asignatura y nivel.

\begin{tabular}{|c|c|c|c|c|c|}
\hline ftem & Matemáticas & $\begin{array}{c}\text { Lengua } \\
\text { y Literatura }\end{array}$ & $\begin{array}{l}\text { Ciencias } \\
\text { Naturaies }\end{array}$ & $\begin{array}{l}\text { Ciencias } \\
\text { Sociales }\end{array}$ & Nivel \\
\hline 11 & 1,35 & 1,56 & 1,35 & 1,72 & \multirow{3}{*}{$1^{\circ} \mathrm{DE}$ BUP } \\
\hline 16 & 1,85 & 2,27 & 1,74 & 2,02 & \\
\hline 20 & 0,97 & $\underline{0.82}$ & 1,31 & 1,07 & \\
\hline 11 & 1.05 & 1,35 & 1,28 & 1,12 & \multirow{3}{*}{ COU-C } \\
\hline 16 & 1.44 & 1,74 & 1,95 & 1,70 & \\
\hline 20 & 1,58 & 1,29 & 1,22 & 1,40 & \\
\hline 11 & 1,11 & 1.46 & 1,11 & 1,10 & \multirow{3}{*}{ COU-L } \\
\hline 16 & 1,84 & 2,06 & 1,66 & 1,68 & \\
\hline 20 & 1,01 & 1,13 & 1,42 & 1,22 & \\
\hline
\end{tabular}

Negrita: Vajores máximos.

Subrayado: Valores mínimos. 
actividades, el rendimiento escolar en términos de aprendizaje sería más alto. En todo caso, como se desprende de los resultados, la planificación de las actividades y tareas de aprendizaje sufre los mismos condicionantes y problemas en las cuatro asignaturas encuestadas; de lo anterior se desprende que nuestro alumnado advierte reiteración y monotonía en las mismas y que declara que son muy poco diversificadas.

\section{B.2.3. Papel del profesorado}

Centremos la atención en los valores medios por niveles; éstos figuran en la tabla XXV.

Los estudiantes de $1^{\circ}$ de BUP consideran a los docentes de Ciencias Sociales flexibles, con valores que están por encima de la media; los estudiantes de COU-Ciencias manifiestan que en Matemáticas se les brindan pocas opciones para trabajar los temas.

En relación con el clima de aula, los valores obtenidos muestran que el alumnado de $1^{\circ}$ de BUP está satisfecho con el clima que generan los docentes de Lengua y Literatura para aprender. COU-Ciencias tiene para Matemáticas una puntuación inferior a la media, mostrando, por tanto, que el profesorado consigue en poca medida el clima adecuado. Paradójicamente es el alumnado de $1^{\circ}$ de BUP el que le ha atribuido a los docentes de Lengua y Literatura el mayor valor en relación con la distancia mantenida con los jóvenes. Existe, pues, una aparente contradicción entre la creación de un clima adecuado por parte de los docentes de Lengua y Literatura y el mantenimiento de una actitud distante con el alumnado. La impresión que captan los estudiantes de este nivel, a juzgar por los resultados, es que un mayor distanciamiento genera un mejor clima de trabajo.

Del mismo modo, resulta curioso que el menor distanciamiento entre estudiantes y docentes lo adviertan los jóvenes de COU-Ciencias en Matemáticas, asignatura en la que habían manifestado que el profesorado conseguía en poca medida mantener el clima adecuado para el aprendizaje.
Parece que se establecen conexiones, en ambos casos, de cordialidad en las relaciones entre profesorado y ahumnado e incapacidad o menor capacitación para crear el clima idóneo para el aprendizaje. Estas relaciones planteadas por los estudiantes pueden entenderse en la medida en que están dando información sobre el tipo de trabajo que lleva a cabo el docente en el aula, el papel que está desarrollando en la misma. En este sentido, un trabajo docente tradicional, centrado en el profesor como eje del mismo, requiere un clima determinado que es el que los estudiantes están captando como normal, en el que existe un mayor distanciamiento entre ambos protagonistas del hecho educativo.

Los resultados obtenidos para Matemáticas muestran que existen diferencias significativas en el ítem 11 entre $1^{\circ}$ de BUP y COU-Ciencias $(99,0)(\mathrm{t}=2,60 ;$ g.l. $=281$; $\mu=0,001)$. En los ítems 16 y 20 , de la misma asignatura, son muy significativamente diferentes $1^{\circ}$ de BUP/COUCiencias $(\mathrm{t}=3,83 ; \mathrm{g} . \mathrm{L} .=280 ; \mu=0)(\mathrm{t}=-5,10 ; \mathrm{g} . \mathrm{l}=282$; $\mu=0)$ y COU-Ciencias / COU-Letras $(\mathrm{t}=-3,66 ; \mathrm{g} . \mathrm{l}$. $=$ $247 ; \mu=0)(\mathrm{t}=4,49 ; \mathrm{g} \cdot 1 .=250 ; \mu=0)$.

En Lengua y Literatura no hay diferencias en el ítem 11 entre los distintos niveles. El ítem 16 muestra diferencias muy significativas entre $1^{\circ}$ de BUP y COU-Ciencias $(99,9)(\mathrm{t}=5,68 ; \mathrm{g} .1 .=267 ; \mu=0)$ y entre COU-Ciencias y COU-Letras $(99,9)\left(\mathrm{t}=-3,24 ; \mathrm{g} . \mathrm{I}_{\mathrm{t}}=243 ; \mu=0,001\right)$.

Ciencias Naturales no presenta diferencias en los ítems 11 y 20 . El ftem 16 muestra una diferencia altamente significativa $(99,7)$ entre ambos $\mathrm{COU}(\mathrm{t}=2,96 ; \mathrm{g} .1$. $=$ $262 ; \mu=0,003)$.

Ciencias Sociales presenta diferencias en los ítems 11 y 16; en ambos son muy significativas entre $1^{\circ}$ de BUP y los dos COU $(99,9$ en el primero y 99,8 en el segundo caso $)(\mathrm{t}=5,17 ; \mathrm{g} . \mathrm{l} \mathrm{l}=245 ; \mu=0)(\mathrm{t}=3,1 \mathrm{I} ; \mathrm{g} . \mathrm{I}=247 ; \mu$ $=0,002)$.

El vaior global que se obtiene del papel del profesorado es de 1,45 , valor que se interpreta como la definición de una tendencia del profesorado que apunta tímidamente a

Tabla XXVI

Valores medios del papel del profesorado por asignatura y nivel.

\begin{tabular}{|c|c|c|c|c|}
\hline & $1^{\circ}$ de BUP & CoU-Ciencias & COU-Letras & $\bar{X}$ \\
\hline MATEMÁTICAS & 1,39 & 1,36 & 1,31 & 1,36 \\
\hline LENGUA Y LITERATURA & 1,57 & 1,46 & 1,55 & 1,53 \\
\hline CIENCIAS NATURALES & 1,45 & 1,49 & 1,40 & 1,45 \\
\hline CIENCIAS SOCIALES & 1,60 & 1,41 & 1,33 & 1,46 \\
\hline
\end{tabular}

Negrita: Valores máximos.

Subrayado: Valores mínimas. 
plantear el trabajo docente en otros términos pero que, según los estudiantes, está en la tónica de la labor profesional tradicional. Por asignaturas, los resultados son los siguientes (Tabla XXVI).

En Ciencias Sociales se observan diferencias entre $1^{\circ} \mathrm{de}$ BUP y ambos COU, que son muy significativas $(99,8$ con COU-Ciencias $(t=3,19 ; \mathrm{g} .1 .=243 ; \mu=0,002)$ y 99,9 con COU-Letras $(t=4,33 ; g .1 . \Rightarrow 230 ; \mu=0)$ ). No se han detectado diferencias en las otras tres asignaturas.

Los valores más bajos en relación con la actividad y el talante del profesorado están representados por Matemáticas y los más altos, por Lengua y Literatura, en valores medios, suponiendo esto una diferencia escasa de 0,17 puntos. Analizando los niveles vemos que son las mismas asignaturas las que tienen los mínimos en COU-Letras (Matemáticas) y máximos en $1^{\circ}$ de BUP (Lengua y Literatura).

\section{B.2.4. Medios y recursos}

Analizamos los datos obtenidos para este elemento de la metodología por asignatura y nivel en la tabla XXVII.

La asignatura en la que más se utiliza el libro de texto en $1^{\circ}$ de BUP es Ciencias Sociales y se hace de forma muy frecuente. Matemáticas es la asignatura que menos usa dichos materiales pero, en todo caso, su frecuencia en este nivel es cercana a «bastante».

El uso de actividades y materiales más diversos viene representado, según el alumnado, por Ciencias Naturales de $1^{\circ}$ de BUP; pero este valor no llega tan siquiera a «bastante». Prácticamente es nulo en Matemáticas de COU-Ciencias.
El tipo de comportamiento viene muy determinado por el profesorado en Ciencias Naturales de $1^{\circ}$ de BUP, siendo en este sentido el valor más bajo el de Lengua y Literatura en COU-Letras.

El ítem 9 no presenta diferencias entre niveles en ninguna de las asignaturas.

En Matemáticas se observan diferencias entre $1^{\circ}$ de BUP y COU.Ciencias, que son muy significativas, tanto en el ítem $14(99,8)(\mathrm{t}=3,13 ; \mathrm{g} .1=281 ; \mu=0,002)$ como en el ítem $19(99,9)(t=6,84 ;$ g.l. $=280 ; \mu=0)$; en éste último también hay diferencias muy significativas entre $1^{\circ}$ de BUP y COU-Letras $(99,9)(\mathrm{t}=6,53 ; \mathrm{g} .1 .=240$; $\mathfrak{l}=0$ ).

Las diferencias entre $1^{\circ}$ de BUP y COU-Letras también son muy significativas para el ítem 14 en Lengua y Literatura $(\mathrm{t}=-3,16 ; \mathrm{g} . \mathrm{I} .=254 ; \mu=0,002)$; entre ambos COU son altamente significativas $(99,7)(\mathrm{t}=-3,05 ; \mathrm{g} . \mathrm{l}=$ $243 ; \mu=0,003)$. En el ítem 19, $1^{\circ}$ de BUP comparado con ambos COU muestra diferencias muy significativas $(99,9$ en ambas relaciones $)(t=4,14 ; g .1 .=262 ; \mu=0)(t=5,48$; g.l. $=253 ; \mu=0$ ).

Ciencias Naturales y Ciencias Sociales muestran diferencias en el ítem 19; $1^{\circ}$ de BUP es significativamente diferente tanto en COU-Letras $(99,9)$ como en COUCiencias $(99,9)$ en ambas asignaturas y con los mismos valores $(t=5,91 ; g . l .=284 ; \mu=0$ para Ciencias Naturales) $(\mathrm{t}=3,59 ; \mathrm{g} .1 .=249 ; \mu=0$ para Ciencias Sociales $)$.

El valor medio como elemento metodológico es 1,73 , lo que representa la poca disponibilidad del profesorado a utilizar vías distintas, mecanismos diferentes, recursos variados, protagonismos compartidos, etc. Observemos

Tabla XXVII

Valores medios de los distintos fiterns de medios y recursos por asignatura y nivel.

\begin{tabular}{|c|c|c|c|c|c|}
\hline Ítem & Matemáticas & $\begin{array}{c}\text { Lengua } \\
\text { y Literatura }\end{array}$ & $\begin{array}{c}\text { Ciencias } \\
\text { Naturales }\end{array}$ & $\begin{array}{l}\text { Ciencias } \\
\text { Sociales }\end{array}$ & Nivel \\
\hline 9 & 1,87 & 2,39 & 2,38 & 2,60 & \multirow{3}{*}{$1^{\circ} \mathrm{DE}$ BUP } \\
\hline 14 & 0,64 & 0,99 & 1.69 & 1,39 & \\
\hline 19 & 2,24 & 1,84 & 2,27 & 2,07 & \\
\hline 9 & 1,9653 & 2,22 & 2,37 & 2,53 & \multirow{3}{*}{ COU.C } \\
\hline 14 & 0,34 & 1,03 & 1,65 & $t, 36$ & \\
\hline 19 & 1,43 & 1,31 & 1,59 & 1,65 & \\
\hline 9 & 2,08 & 2,36 & 2,55 & 2,55 & \multirow{3}{*}{ COU.L } \\
\hline 14 & 0,58 & 1,38 & 1,59 & 1,67 & \\
\hline 19 & 1,40 & 1,13 & 1,57 & 1,57 & \\
\hline
\end{tabular}


Tabla XXVIII

Resultados medios de los recursos por asignatura y nivel.

\begin{tabular}{|c|c|c|c|c|}
\hline & $1^{\circ} \mathrm{de}$ BUP & COU-Ciencias & CoU-Letras & $\bar{X}$ \\
\hline MATEMÁTICAS & 1,59 & 1,25 & 1,35 & 1,39 \\
\hline LENGUA Y LITERATURA & 1,74 & 1,51 & 1,63 & 1,90 \\
\hline CIENCIAS NATURALES & 2,12 & 1,87 & 1,63 \\
\hline CIENCIAS SOCIALES & 2,02 & 1,93 & 1,96 \\
\hline
\end{tabular}

Negrita: Valores máximos.

Subrayado: Valores mínimos.

la tendencia de este elemento de la metodología por niveles (Tabla XXVIII).

En Matemáticas, $1^{\circ}$ de BUP y COU-Letras son altamente diferentes $(99,6)(\mathrm{t}=2,91 ; \mathrm{g} .1 .=233 ; \mu=0,004)$ y $1^{\circ}$ de BUP en comparación con COU.Ciencias es muy significativamente diferente $(t=5,18 ; g .1=276 ; \mu=0)$. Esto último ocurre también en Lengua y Literatura y en Ciencias Naturales. En esta última asignatura las diferencias entre $1^{\circ}$ de BUP y COU-Letras son significativas $(99,4)(\mathrm{t}=2,78 ; \mathrm{g} .1 .=253 ; \mu=0,006)$, así como entre $1^{\circ}$ de BUP y COU-Ciencias en Ciencias Sociales $(99,2)$ $(\mathrm{t}=2,66 ; \mathrm{g} .1 .=245 ; \mu=0,008)$.

Paradójicamente en relación con sus posibilidades, por una parte, y con el interés y gusto del alumnado, por otra, las Ciencias Naturales ostentan el valor más alto tanto en medias como por niveles, con la única excepción de COU-Letras, nivel en el que es superado por Ciencias Sociales sólo en tres centésimas. El valor más bajo lo tiene Matemáticas de COU.Ciencias y las Matemáticas en general.

Tabla XXIX

Valores medios de los ítems de entorno de aprendizaje por asignatura y nivel.

\begin{tabular}{|c|c|c|c|c|c|}
\hline Ítem & Matemáticas & $\begin{array}{c}\text { Lengua } \\
\text { y Literatura }\end{array}$ & $\begin{array}{l}\text { Ciencias } \\
\text { Naturales }\end{array}$ & $\begin{array}{l}\text { Ciencias } \\
\text { Sociales }\end{array}$ & Nivel \\
\hline 8 & 0,50 & 0,81 & 0,91 & 0,95 & \multirow{3}{*}{$I^{\circ} \mathrm{DE}$ BUP } \\
\hline 13 & 1,94 & 2,13 & 2,13 & 2,00 & \\
\hline 18 & 1,89 & 1,85 & 2,29 & 1,85 & \\
\hline 8 & 0,20 & 0,82 & 1.36 & 1,20 & \multirow{3}{*}{ COUC } \\
\hline 13 & 2,16 & 2,26 & 2,17 & 2,21 & \\
\hline 18 & 1,82 & 2,03 & 2,10 & 1,98 & \\
\hline 8 & 0,55 & 1,26 & 1,42 & 0,49 & \multirow{3}{*}{ COUL } \\
\hline 13 & 2,07 & 2,15 & 2,25 & 2,17 & \\
\hline 18 & 1,79 & 1,85 & 2,19 & 1,85 & \\
\hline
\end{tabular}




\section{B.2.5. Entorno de aprendizaje}

Se presentan a continuación los datos referidos por asignaturas y niveles para este aspecto de la metodología (Tabla XXIX).

El valor más bajo en relación con la organización en grupos es el que ha asignado el alumnado a Matemáticas en $\mathrm{COU}$-Ciencias.

La mayor dirección en la organización del tiempo se presenta en Lengua y Literatura en COU-Ciencias.

La distribución del espacio en filas presenta una diferencia entre el valor más alto y el más bajo de 0,5 puntos, to que demuestra la poca dispersión de estos datos. Es más tradicional (en fílas) en Ciencias Naturales de $1^{\circ}$ de BUP y menos, aun siendo alta, en Matemáticas de COULetras.

Revisemos las diferencias por asignaturas entre distintos niveles: los ítems 13 y 18 no muestran discrepancias en ninguna asignatura. Para el ítem 8, COU-Ciencias muestra diferencias muy significativas tanto con $1^{\circ}$ de BUP $(\mathrm{t}=3,81 ; \mathrm{g} .1 .=279 ; \mu=0)$ como con COU-Letras $(t=-4,02 ; g . l .=248 ; \mu=0)$ en Matemáticas $(99,9$ en ambos casos). Lo mismo ocurre en Lengua y Literatura. En Ciencias Naturales, $1^{\circ}$ de BUP es muy significativamente diferente tanto con COU-Letras como con COU. Ciencias ( 99,9 en cada una de las dos comparaciones) $(\mathrm{t}=-3,77 ; \mathrm{g} . \mathrm{l} .=285 ; \mu=0)(\mathrm{t}=-4,14 ; \mathrm{g} .1 .=256 ; \mu=0)$. COU-Letras es el nivel que, en el caso de Ciencias Sociales, muestra diferencias con los otros dos niveles.

La media calculada para este elemento metodológico en su conjunto es de 1,65 , valor que se considera relativamente alto en relación con planteamientos alternativos relacionados con agrupamientos más flexibles de los estudiantes, con una organización del espacio más idónea para la interacción entre ellos y con una distribución y organización del tiempo más acorde con las tareas que se asignen.
Por niveles, los resultados son los que se indican en la tabla XXX.

El valor más alto, en medias, lo presenta Ciencias Naturales, lo que viene a suponer el planteamiento más tradicional a este respecto. El valor más bajo, dentro de un estrecho margen de variación, es el que el alumnado asigna a Matemáticas. Por niveles, son también Ciencias Naturales (COU-Letras) y Matemáticas (COU-Ciencias), los que tienen los valores más alto y más bajo.

Las diferencias entre $1^{\circ}$ de BUP y COU-Ciencias son en Ciencias Sociales altamente significativas $(t=-2,82 ; \mathrm{g} .1$. $=238 ; \mu=0,005$ ) y entre este último y COU.Letras muy significativas $(t=3,68 ; g . l,=223 ; \mu=0)$. No se observan más diferencias entre niveles en las otras tres asignaturas.

\section{B.3. Valoración conjunta de la metodología}

Una vez que hemos presentado los valores pormenorizados de cada uno de los elementos de la metodología, conviene que analicemos de forma global lo que de ellos se deriva; es decir, si cabe, la metodología o tipo de trabajo que se desarrolla en las aulas. De esta valoración conjunta resulta la tabla XXXI.

A la vista de estos resultados, las cuatro asignaturas mantienen con «bastante» frecuencia estrategias expositivas; la diversificación y el desarrollo de diferentes actividades y tareas es "poca»; el profesorado es relativamente flexible y cercano a los estudiantes; la utilización de medios y recursos diferentes no llega a ser «bastante» variada; y, por último, la organización del entorno es cercana a «bastante» tradicional.

De lo anterior se deriva que el trabajo docente en las distintas asignaturas adolece de planteamientos innovadores, que no se han incorporado a las prácticas pedagógicas los principios y las pautas que la teoría curricular ha ido aportando con objeto de mejorar los

Tabla XXX

Valores globales de entorno de aprendizaje por asignatura y nivel.

\begin{tabular}{|l|c|c|c|c|}
\hline & $1^{\circ}$ de BUP & COU-Ciencias & CoU-Letras & $\bar{X}$ \\
\hline MATEMÁTICAS & 1,45 & $\frac{1,40}{1,71}$ & 1,46 & $\frac{1,43}{1,75}$ \\
\hline LENGUA Y LITERATURA & 1,60 & 1,75 & 1,68 \\
\hline CIENCIAS NATURALES & 1,77 & 1,95 & $\mathbf{1 , 8 6}$ \\
\hline CIENCIAS SOCIALES & 1,59 & 1,79 & 1,50 & 1,63 \\
\hline
\end{tabular}

Negrita: Valores máximos.

Subrayado: Valores minimos. 
Tabla XXXI

Valores obtenidos para los diferentes elementos de la metodología por asignatura.

\begin{tabular}{|c|c|c|c|c|c|}
\hline & Matemáticas & $\begin{array}{c}\text { Lengua } \\
y \text { Literatura }\end{array}$ & $\begin{array}{l}\text { Ciencias } \\
\text { Naturales }\end{array}$ & $\begin{array}{l}\text { Ciencias } \\
\text { Sociales }\end{array}$ & Nivel \\
\hline ESTRATEGIAS & 2,15 & 2,15 & $\underline{2.09}$ & 2,15 & 2,13 \\
\hline ACTIVIDADES Y TAREAS & 1,24 & 1,34 & $\underline{1,1 I}$ & 1,19 & 1,22 \\
\hline PAPEL DEL PROFESORADO & 1,36 & 1,53 & 1,45 & 1,46 & 1,45 \\
\hline MEDIOS Y RECURSOS & $\underline{1,39}$ & 1,63 & 1,96 & 1,93 & 1,73 \\
\hline ENTORNO DE APRENDIZAJE & $\underline{1.43}$ & 1,68 & 1,86 & 1,63 & 1,65 \\
\hline
\end{tabular}

Negrita: Valores máximos

Subrayado: Valores mínimos.

procesos de enseñanza y los de aprendizaje del alumnado, su rendimiento escolar y el clima en el que desarrollarlos.

Analizando estos datos por asignaturas parece desprenderse de los resultados que es el profesorado de Ciencias Naturales el que desarrolla un trabajo más tradicional, más cercano a los patrones habitualmente considerados del aula, si bien, las diferencias, como se ve, no son significativas.

Si observamos los aspectos metodológicos (los cinco elementos de la metodología) por centros, Ios resultados obtenidos en el IB-A muestran diferencias muy significativas entre las cuatro asignaturas. El entorno es muy significativamente diferente entre las cuatro materias; esta diferencia es altamente significativa entre Matemáticas y Ciencias Sociales. Se observan diferencias muy significativas también entre Lengua y Literatura, y Ciencias Naturales relativas a actividades y tareas.

El IB-B muestra diferencias altamente significativas $(99,6 \%)$ en el papel del profesorado entre las diferentes asignaturas. El entorno de aprendizaje es significativamente diferente, también, entre todas las asignaturas, lo que quiere decir que en las cuatro disciplinas se organiza el espacio, el tiempo y al alumnado de manera diferente, ya que se capta de formas muy distintas por parte del alumnado. Los medios y recursos muestran una diferencia muy significativa $(99,9 \%)$ de Matemáticas con respecto a Ciencias Naturales y a Ciencias Sociales y de Lengua y Literatura con respecto a Ciencias Naturales y a Ciencias Sociales. Las actividades tienen una diferencia muy significativa entre Ciencias Naturales y Ciencias Sociales; los estudiantes captan de manera muy significativamente diferente las actividades que se rea-

Tabla XXXII

Valores medios del interés, valores intrínsecos y diferencias por asignatura.

\begin{tabular}{|c|c|c|c|c|}
\hline & Matemáticas & Lengua y Literatura & Ciencias Naturales & Ciencias Sociales \\
\hline VALOR MEDIO (A) & 2,83 & 3,00 & 2,95 & 2,72 \\
\hline VALOR INTRÍNSECO & 2,36 & 2,19 & 2,58 & 2,39 \\
\hline DIFERENCIA & 0,47 & 0,81 & 0,37 & 0,33 \\
\hline
\end{tabular}

Negrita: Valores máximos.

Subrayado: Valores mínimos. 
lizan en las clases de Ciencias Naturales y de Ciencias Sociales.

En el IB-C el papel del profesorado muestra diferencias altamente significativas $(99,5 \%)$ entre Matemáticas, y Lengua y Literatura. El entorno de aprendizaje es muy significativamente diferente $(99,9 \%)$ si comparamos Matemáticas con Lengua y Literatura y con Ciencias Naturales. Las actividades y tareas presentan también una diferencia muy significativa entre Lengua y Literatura y las otras tres asignaturas. Los medios y recursos muestran también diferencias muy significativas en todas las asignaturas exceptuando Lengua y Literatura y Ciencias Naturales.

Con los resultados expuestos, la segunda hipótesis, relativa a un trabajo docente más innovador en la enseñanza de las Ciencias Naturales queda refutada, pues, analizados los mismos, se puede ver que en esta asignatura se observa por parte del alumnado una metodología de corte conservador, incluso más tradicional que en las otras asignaturas en muchos aspectos.

\section{Relación entre interés y aspectos metodologógicos}

Con objeto de analizar en qué medida los intereses encontrados para las distintas asignaturas se relacionan con el tipo de trabajo docente desarrollado en las mismas, desde la óptica del alumnado, se ha realizado el cálculo de los coeficientes de regresión múltiple de la variable dependiente «interés» frente a las variables representadas por los distintos elementos de la metodología. De esta manera, se han calculado los valores intrínsecos de cada una de las cuatro asignaturas o, Io que es lo mismo, la medida del interés que muestran los estudiantes independientemente de to que se desarrolla o hace en los centros escolares. Del mismo modo, se han obtenido las diferencias entre estos valores y los que, una vez contemplada la escolarización, se han reflejado en la parte A del cuestionario. Cabe comentar, sin embargo, que es difícil delimitar si estos valores intrínsecos son sólo producto de los individuos en cuestión o si su historia académica anterior juega algún papel en los intereses personales, al margen de la escuela. No se ha considerado necesario tener en cuenta en este cálculo de regresión las variables nivel y centro. Los datos obtenidos figuran en la tabla XXXII.

Como vemos, las diferencias son bastante bajas, lo que viene a representar que el trabajo que se desarrolla en las aulas, en definitiva, la labor docente, tiene una baja o poca influencia en el interés mostrado por el alumnado. Es evidente que, como docentes, no estamos consiguiendo aumentar este interés en una medida aceptable, no estamos transmitiendo la utilidad de lo que enseñamos o, cuanto menos, nuestros estudiantes no lo captan, como ya se ha planteado (Yager y Penick, 1984; Carrascosa, 1991).

Si analizamos cada una de las asignaturas, el estudio de regresión muestra cuáles son los elementos de la meto- dología que mayor influencia tienen en el aumento del interés del alumnado.

\section{C.I. Matemáticas}

Como responsables del aumento en el interés $(0,47)$, una vez se ha producido escolarización, figuran en primer lugar el papel del profesorado en un $41 \%$ y las actividades y tareas que se desarrollan en un $34 \%$. Resulta curioso que el papel del profesorado sea lo que más influencia tiene en el aumento detectado, por cuanto la valoración que se ha hecho desde esta asignatura para este elemento es baja ( 1,36 en la tabla 31$)$; la diversificación de actividades y tareas tampoco tiene una valor alto $(1,24)$. El uso de medios y recur'sos variados muestra una curiosa peculiaridad: influye en un $24 \%$ pero negativamente, lo que se interpreta como las preferencias del alumnado por la utilización de pocos o de un solo tipo de materiales. Es una realidad, que la práctica docente corrobora, que los jóvenes se muestran más cómodos y les resulta más fácil que sus docentes, en esta asignatura, reproduzcan o repitan un mismo patrón con los mismos materiales.

\section{C.2. Lengua y Literatura}

La mayor diferencia detectada entre el valor intrínseco y el que se había manifestado a través de la escolarización es la que muestra esta asignatura $(0,81)$. Son responsables de este aumento en el interés las actividades y tareas $(58 \%)$ y el papel del profesorado $(41 \%)$. Como se ve en la tabla XXXI, estos valores son los máximos si comparamos con el resto de las asignaturas, si bien en ambos casos, rondan el 1,5, lo que supone aproximadamente la mitad de la escala establecida que, como se recordará, tiene cuatro niveles $(0,1,2,3)$.

\section{C.3. Ciencias Naturales}

Esta asignatura muestra una diferencia bastante baja $(0,37)$, diferencia mínima que consigue la escolarización. La situación descrita por el alumnado en relación con el trabajo que los docentes de Ciencias Naturales desarrollamos en el aula es evidente que no favorece o potencia una ciencia y un trabajo científico como «empresa vital y fascinante" (Schibecci, 1986).

La mínima diferencia detectada se debe casi en su totalidad a los medios y recursos como elemento de la metodología, hecho este que parece evidente y que se corrobora con el dato obtenido en la tabla XXXI $(1,96)$.

\section{C.4. Ciencias Sociales}

La diferencia que se ha encontrado en este caso es la más baja $(0,33)$. Las actividades y tareas propuestas son en su totalidad las responsables de este mínimo aumento, si bien su consideración global $(1,19)$ (Tabla XXXI) es baja; recordemos que la escala de valoración varía entre 0 y 3 . Un aumento en la frecuencia y en la diversificación de las actividades es de suponer que influiría positivamente en el aumento del interés del alumnado por esta 
asignatura, ya que se ha mostrado como el elemento más responsable de dicho aumento.

Como se ha expuesto, se observa que hay una relación directa entre el aumento del interés mostrado por los estudiantes y algunos de los elementos de la metodología utilizada en cada una de las asignaturas. La ausencia de los restantes elementos no debe interpretarse en el sentido de que no tienen ninguna influencia, pues lo que viene a representar es que, comparativamente, su influencia es muy poco significativa.

\section{DISCUSIÓN Y CONCLUSIONES}

1) De los datos globales obtenidos se desprende que la primera de las hipótesis, relativa al mayor interés del alumnado por Ciencias Naturales, por los temas que se estudian queda refutada, ya que Lengua y Literatura obtiene, en virtud de los temas incluidos en dicha asignatura, una puntuación mayor en 0,05 décimas si atendemos a los valores medios. Cabe, sin embargo, comentar que Ciencias Naturales es la asignatura que muestra la mayor dispersión, por una parte, y, por otra, ostenta los temas más valorados en primer y segundo lugar. Por niveles, se confirma la hipótesis tanto para $1^{\circ}$ de BUP como para COU-Ciencias. La posición que ocupa esta asignatura en relación con las demás es intermedia o central en los tres centros.

No se replican claramente los datos encontrados por Yager y Penick (1986) en relación con la pérdida del interés por, Ciencias Naturales a medida que aumenta la escolaridad, si bien no ha sido éste un propósito expreso del trabajo desarrollado.

Los estudiantes no advierten diferencias significativas en los modos de trabajar de los docentes de las distintas asignaturas; sin embargo, parece indicarse que es en Ciencias Naturales donde se dan los planteamientos más tradicionales. Esta afirmación supone la refutación de la segunda de las hipótesis planteadas.

2) Yager y Penick (1984) plantean la necesidad de redefinir los objetivos de la enseñanza, ya que los estudiantes, en una gran mayoría, sienten que no participan en la selección del contenido objeto de su aprendizaje. De hecho, de los datos se deriva que los temas seleccionados en las cuatro asignaturas resultan poco atractivos para los mismos, pues solamente se iguala o supera el valor 3 («Bastante interés») en Matemáticas (COULetras) y Lengua y Literatura (COU-Letras). El análisis interno elaborado para cada una de las asignaturas refleja la necesidad de que los docentes planteemos una reflexión seria sobre el contenido seleccionado en las mismas y sobre la forma de hacerlo llegar al alumnado.

Parece difícil aceptar en ta actualidad que se desarrolle en los estudiantes un aprendizaje, un conocimiento en suma, descontextualizado e inútil, que no les permita dar solución o enfrentarse a los problemas con los que se encuentren. Si consideramos el conocimiento como un conjunto de saberes científicamente aceptados, socialmente construidos, sea en la disciplina que sea, parece esencial llevar a cabo una democratización de la educación cientifica que tenga en cuenta las necesidades de especificos grupos sociales (Layton, Davey y Jenkins, 1986, citados por Moreno, 1987, pp. 161).

3) Si consideramos la valoración que el alumnado hace de la labor docente como un instrumento de evaluación (Carrascosa et al., 1991), estas opiniones adquieren una importancia fundamental a la hora de planificar las propuestas didácticas. La necesidad de cambio en el trabajo del profesorado en Ias asignaturas objeto de estudio ya se había detectado y había sido expresada por los estudiantes (Afonso López et al., 1991), pero no se habían explicitado las líneas que se deberían seguir en la reflexión que propicie dicho cambio. Analizando los resultados, se observa que existen lagunas importantes en la consideración de los diferentes elementos de la metodologia, lo que muestra una manifiesta incoherencia, pues las decisiones que afectan a uno de ellos son inconsistentes, e incluso contradictorias, con las que se deciden para el resto. La situación descrita, si bien desde la perspectiva del alumnado, muestra un trabajo muy poco innovador si tenemos en cuenta las líneas que se han abierto en los últimos años relativas a los procesos de enseñanza y de aprendizaje en las cuatro asignaturas. En este sentido, debe tenerse en cuenta que la escuela y particularmente el profesor pueden jugar, a pesar de un medio desfavorable, un papel muy importante debido a su gran potencialidad para conseguir que los alumnos progresen (Simpson y Oliver, 1990, citados por Carrascosa, 1990, p. 174). Es evidente que toda la responsabilidad no recae sólo en el profesorado, ya que se trata de un sistema muy complejo en el que intervienen otros muchos factores, pero que, como muestran también otras investigaciones, el papel de los docentes y su preparación en los aspectos cuestionados es ineludible.

4) No es de extrañar que autores como Yager y Penick (1984,1986) hayan encontrado resultados desfavorables hacia la ciencia, en particular; en el sentido de que, a medida que aumenta la escolaridad, la actitud y la percepción de la misma es más negativa, dato este que no tiene un correlato exacto en esta investigación si el trabajo que se desarrolla en las aulas es percibido de la manera que los resultados obtenidos muestran. En estas condiciones, si bien hemos de tener en cuenta que se trata del punto de vista del alumnado, es evidente que, en todo caso, se hace necesaria una reflexión profunda de los docentes que aborde estos temas. No obstante, hemos de tener en cuenta que la percepción que tienen los estudiantes de sus profesores también se vuelve más negativa a medida que aumenta la escolaridad, según muestran, en relación con los docentes de ciencias en sentido estricto, Yager y Penick (1986).

5) Los distintos elementos de la metodología guardan una relación muy estrecha entre sí, de tal manera que la forma en que cada docente los contemple puede llegar a definir diferentes modelos de profesorado. Una determinada opción decidida sobre la estrategia a utilizar debe mantener coherencia con las decisiones que esto implica 
en términos de las actividades que se hagan, los medios y los recursos que se usen en las mismas, el papel que corresponde hacer o asumir a dicho docente y la manera de organizar el entorno de aprendizaje. En este sentido, los datos obtenidos resultan curiosos, pues, como se ha expuesto, en ninguno de los casos se observa esta coherencia y sí cuál o cuáles son los elementos que se han visto primados, aunque con valores bajos casi en todos los casos, y son responsables de los pocos incrementos detectados en el interés del alumnado por las distintas asignaturas.

Lo que los estudiantes nos están diciendo es que no estamos potenciando una visión del conocimiento científico (globalmente considerado y, por lo tanto, el conocimiento generado en todas y cada una de las asignaturas) ajustada a la realidad actual ni formal ni pedagógicamente. ¿Cómo vamos a conseguir que la adquieran? ¿Cómo vamos a obtener que se produzcan esos aprendizajes? ¿Cómo vamos a lograr que estos aprendizajes y la forma de enseñarlos aumenten el interés por las asignaturas, por el propio conocimiento?

6) Analizando la bibliografía consultada, se observa que existe una relación directa entre las actitudes de los estudiantes frente a las distintas asignaturas y los resultados académicos. Las percepciones que ellos tienen de los procesos de enseñanza influyen claramente en las actitudes que generan (Schibecci y Riley, 1986). De hecho, entre otras variables analizadas, Talton y Simpson (1986) constatan que las que están relacionadas con el ambiente educativo son las que ejercen una influencia mayor en la actitud generada por los estudiantes hacia su aprendizaje. Los resultados presentados relativos a la influencia de los aspectos metodológicos en los intereses expresados para las diferentes asignaturas estarían en la línea de lo expuesto, ya que coinciden con estas apreciaciones.

$\mathrm{Si}$, como señalan Simpson y Oliver (1990), el interés y los sentimientos hacia la ciencia (hacia los conocimientos o el conocimiento científico) se ven mayoritariamente determinados por lo que se hace en la escuela, los resultados obtenidos muestran la gravedad de la situación descrita en el sentido de que el trabajo docente que se está llevando a cabo en las aulas no está potenciando un aprenđizaje eficaz de las distintas disciplinas. Sin el desarrollo adecuado de estas actitudes, tarea primordial del profesorado, no se van a producir los aprendizajes planificados, ya que ésta es una variable de gran influencia en los mismos.

7) Los resultados obtenidos ofrecen una abundante y útil información, lo que demuestra que las opiniones del alumnado constituyen un instrumento eficaz de evaluación del trabajo docente, si bien es evidente que no debe ser el único. El análisis de los datos presenta una descripción de lo que ocurre en las aulas de las cuatro asignaturas consideradas que es particularmente rica en el sentido de que muestra las líneas que deberían seguirse con objeto de evitar, en la implantación y generalización de Ia LOGSE, que se repitan o reproduzcan las situaciones que se han presentado. Como queda de manifiesto, está constituyendo un escollo importante la selección del contenido que ha adolecido de un proceso democrático, tanto para estudiantes como para docentes, y que no responde a los intereses y necesidades de las personas a las que va destinado, con lo que difícilmente podemos motivarlos para su aprendizaje. La LOGSE a priori también muestra una selección, pero supuestamente más flexible y negociada, si bien es cierto que establece lo que se ha dado en Ilamar currículo mínimo a través de los decretos de contenidos mínimos que dictan lo que todo docente debe garantizar en su alumnado. Lo que sí queda en manos del profesorado es la organización de dicho contenido en la medida en que los ciclos formativos son más largos, hecho este que puede paliar en cierta medida el problema que, desde nuestro punto de vista, se repetirá en mayor o en menor medida que la expuesta en la investigación realizada.

El otro gran probiema detectado, a través de las opiniones de nuestros estudiantes, está relacionado con la forma de trabajar esos contenidos, con la forma de potenciar su aprendizaje; en definitiva, con la metodología. La LOGSE no debe suponer sólo el cambio formal de la estructura del sistema educativo sino que, lo más importante, debe suponer un profundo cambio en los modos de hacer, de trabajar en los centros, de organizarlos, etc. En este sentido, la apuesta más importante debe centrarse en el profesorado, y es lo que los jóvenes nos están diciendo: en su capacitación, en dotarlo de los instrumentos y herramientas necesarias para poder abordar esta nueva forma de ver los procesos de enseñanza y de aprendizaje. Y es aquí donde el riesgo es mayor porque, de no tomar conciencia de esto, no se van a producir cambios en la labor profesional docente, de manera que los resuItados expuestos en este trabajo se reproducirían en un futuro no muy lejano. Es esencial, pues, que los datos aportados por los estudiantes sobre el trabajo que sus docentes realizan reviertan en una mejor preparación que evite que estas situaciones puedan volver a repetirse. Las decisiones que el profesorado toma en relación con la forma de contemplar los diferentes elementos de la metodología responden a lo que piensa de la enseñanza y a sus marcos teóricos de referencia. Si éstos no son modificados, independientemente y al margen de que las normativas establezcan nuevas formas, no se producirá cambio alguno en sus prácticas docentes. Esto supone, o debería suponer, una apuesta fuerte por la formación del profesorado que, de no hacerse, supondrá, como ya se oye, un gran fracaso para la LOGSE, a pesar de su potente fundamentación teórica.

8) Suponiendo que las percepciones de los estudiantes sobre su instrucción son indicadores válidos de la conducta de los profesores [...], lo que éstos hacen en clase si que produce diferencias en las actitudes y resultados académicos de los alumnos. Los profesores [...] Ino pueden permitirse el lujo de prescindir de las actitudes de los alumnos. El profesor [que asi actúa] puede estar haciendo unflaco servicio a sus estudiantes, haciendo la instruccion menos efectiva de lo que podría ser. Los profesores que exhiben conductas de enseñanza que animen a los estudiantes a ser creativos tienen mayores probabilidades de tener influencia positiva en las actitudes de sus alumnos. 
Estas actitudes, a su vez, pueden tener positiva influencia sobre los resultados académicos (Schibecci y Riley, 1986, citados por Moreno, 1987, p. 251).

Esta cita expresa con total rotundidad la reflexión que se deriva đe los resultados obtenidos así como de las líneas

\section{REFERENCIAS BIBLIOGRÁFICAS}

ACEVEDO DÍAZ, J.A. (1993). Actitudes hacia el aprendizaje de las ciencias físicas, naturales y matemáticas en BUP y COU. Un estudio sobre tres dimensiones. Actas del IV Congreso Internacional sobre Investigación en la Didáctica de las Ciencias y de las Matemáticas, pp. 13-14.

ACEVEDO DÍAZ, J.A. (1993). ¿Qué piensan los estudiantes sobre Ia ciencia? Un enfoque CTS. Actas del IV Congreso Internacional sobre Investigación en la Didáctica de las Ciencias y de las Matemáticas, pp. 11-12.

AFONSO LÓPEZ, R., BAZO GONZÁLEZ, C., LÓPEZ HERNÁNDEZ, M., MACAU FÁBREGA, M.D. y RODRÍGUEZ PALMERO, M.L. (1991). Los intereses del alumnado y la enseñanza de las ciencias naturales en BUP y COU. Qurriculum, 3, pp. 91-106.

ARANA PÉREZ, J., ESCUDERO ESCORZA, T., GARCÉS CAMPOS, R y PALACIOS GARCÍA, E. (1987). Imagen de las asignaturas de ciencias en la transición de la educación básica a la secundaria. Enseñanza de las Ciencias, 5(1), pp. $10 * 15$.

BOYER, R. y TIBERGHLEN, A. (1989). Las finalidades de la enseñanza de la física y la química vistas por los profesores y alumnos franceses. Enseñanza de las Ciencias, 7(3), pp. 213-222.

BUSQUETS, M.D., CAMPOS, M., FERNÁNDEZ, T.,LEAL, L., MORENO, M. y SASTRE, G. (I993). Los temas transversales. Madrid: Aula XXI. Santillana.

CARRASCOSA ALIS, J. (1985). Reseña bibliográfica de: Yager, E.R. y Penick, J.E. (1984). What students say about science teaching and science teachers. Science Education, 68(2), pp. 134-152. Enseñanza de las Ciencias, 3(1), pp. $57-58$.

CARRASCOSA ALIS, J. (1990). Reseña bibliográfica de Simpson, R. y Oliver, S. (1990). A summary of major influence of attifudes toward an achivement in Science among adolescent students. Science Education, 74(1), pp. 1-18. Enseñanza de las Ciencias, 8(2), pp. 173-174.

CARRASCOSA, J., FERNÁNDEZ, I., GIL, D. y OROZCO, A. (1991). La visión de los alumnos sobre lo que el profesorado de ciencias ha de saber y saber hacer. Investigación en la Escuela, 4.

ESPINOSA, J. Y ROMÁN, T. (1991). Actitudes hacia la ciencia y asignaturas pendientes: dos factores que afectan al rendimiento en ciencias. Enseñanza de las Ciencias, $9(2)$, pp. 151-154.

ESPINOSA GARCÍA, J. y ROMÁN GALÁN, T. (1993). Actitudes hacia la ciencia en estudiantes universitarios de ciencias. Enseñanza de las Ciencias, 11 (3), pp. 297-300. de actuación que necesariamente habrán de abrirse con objeto de mejorar la situación descrita, situación que parece coincidir con otros análisis similares realizados en contextos pedagógicos aparentemente diferentes al de este estudio.

FONTES COSTA, M.A. (1990). Evaluación de valores y actitudes de los alumnos del curso secundario unificado. Un estudio en la disciplina de biología. Enseñanza de las Ciencias, 8(2), pp. 153-156.

GIL PÉREZ, D. (1991). ¿Qué hemos de saber y saber hacer los profesores de ciencias? Enseñanza de las Ciencias, 9(1), pp. 69-77.

MOREIRA, M.A. (1993). La teoría de educación de Novak y el modelo de enseñanza-aprendizaje de Gowin. Fasciculos del CIEF, Serie Enseñanza-Aprendizaje, 4. (Traducción de Marta Pesa, Argentina).

MORENO, M.D. (1987). Reseña bibliográfica de Layton, D., Davey, A. y Jenkins, E. (1986). Science for specific social purposes (SSSP): perspectives on adult scientific literacy. Stadies in Science Education, 13, pp. 27-52. Enseñanza de las Ciencias, 5(2), pp. 159-161.

MORENO, M.D. (1987). Reseña bibliográfica de Schibecci, R.A. y Riley, J.P. (1986). Influence of students background and perceptions on science attitudes and achievement. Journal of Research in Science Teaching, 23(3),pp. 177-187. Enseñanza de las Ciencias, 5(3), pp. 250-251.

MORENO, M.D. (1987). Reseña bibliográfica de Schibecci, R.A. (1986). Images of science and scientists and science education. Science Education, 70(2), pp. 139-149. Enseñanza de las Ciencias, 5(3), pp. 251.

MORENO, M.D. (1987). Reseña bibliográfica de Talton, E.L. y Simpson, R.D. (1986). Relationships of attitudes toward self, family and school with attitude toward science among adolescents. Science Education, 70(4),pp. 363-374. Enseñanza de las Ciencias, 5(3), pp. 249-250.

MORENO, M.D. (1987). Reseña bibliográfica de Yager, E.R. y Penick, J.E. (1986). Perceptions of four age groups toward science class teachers and the values of science. Science Education, 70(4), pp. 335-363. Enseñanza de las Ciencias, 5(3), pp 247-248.

NOVAK, J.D. (1981). Uma teoria de educação. Sao Paulo, pioneira. Traducción de Moreira, M.A. (1977). A theory of education. Comell Univerity Press.

ORTEGA RUIZ, P., SAURA SOLER, J.P., MINGUEZ VALLEJOS, R., GARCÍA DE LAS BAYONAS CAVAS, A., MARTÍNEZ MARTÍNEZ, D. (1992). Diseño y aplicación de una escala de actitudes hacia el estudio de las ciencias experimentales. Enseñanza de las Ciencias, 10(3), pp. 295-303.

SCHWAB, J. (1973). The practical 3: traslation into curriculum. School Review, 81(4), pp. 501-522.

SERRANO, T. (1988). Actitudes de los alumnos y aprendizaje de las Ciencias. Un estudio longitudinal. Investigación en la Escuela, 5, pp. 29-38. 


\section{ANEXO I}

Relación de ítems destinados a la metodología según el orden en el que han sido presentados al alumnado.

\section{ÍTEMS}

Núm. 7: El profesor selecciona materiales y actividades para que los alumnos aprendan.

E

Núm. 8: Para desarrollar el trabajo nos distribuimos en grupos.

EA

Núm. 9: Para aprender utilizo las explicaciones del profesor y el libro de texto. M/R

Núm. 10: En clase se desarrollan debates, discusiones, puestas en común. $\quad$ A/T

Núm. 11: El profesor es flexible, ya que te ofrece distintas opciones para trabajar un tema. PP

Núm. 12: El profesor es el protagonista de la clase, porque es el que explica los temas. E

Núm. 13: El profesor determina el tiempo dedicado a lo que hacemos en clase. EA

Núm. 14: El profesor nos ofrece distintas actividades y materiales (vídeos, diapositivas,
libros, artículos).

Núm. 15: El tiempo en clase fundamentalmente se dedica a realizar actividades. $\quad$ A/T

Nám. 16: El profesor consigue mantener un clima apropiado para que aprenda. $\quad$ PP

Núm. 17: Para aprender llevas a cabo investigaciones sobre cuestiones que han surgido en la
discusión entre todos (tus compañeros, tu profesor y tú).

Núm. 18: Generalmente las mesas están colocadas en filas. $\quad$ EA

Núm. 19: El profesor te indica cómo has de comportarte en su clase. $\quad$ M/R

Núm, 20: El profesor es distante con sus alumnos. $\quad$ PP

E: estrategias; A/T: actividades y tareas; PP: papel del profesorado; M/R: medios y recursos; EA: entorno de aprendizaje. 